\title{
Fractal analysis of directional permeability of gas shale fracture networks: a numerical study
}

Richeng LIU (1),2), Liyuan YU ${ }^{1)}$, Yujing JIANG ${ }^{2), 3) *}$

1) State Key Laboratory for Geomechanics and Deep Underground Engineering, China University of Mining and Technology, Jiangsu 221116, China

2) School of Engineering, Nagasaki University, Nagasaki 8528521, Japan

3) State Key Laboratory of Mining Disaster Prevention and Control Co-founded by Shandong Province and the Ministry of Science and Technology, Shandong University of Science and Technology, Qingdao 266590, China

Corresponding author: Yujing Jiang (Y. Jiang)

Tel: +81-95-819-2612

E-mail address: jiang@nagasaki-u.ac.jp 
Abstract: Since the directional permeability of gas shale fracture networks is significantly dependent on the geometric properties of fractures, in this work, a numerical study was performed to analyze the relationships of them, in which fracture length followed a fractal distribution and fracture orientation followed a normal distribution. Assumptions were made that fractures are more permeable than shale matrix and gas shale fracture networks are consisted of two sets of fractures with different distributions of length, aperture and orientation. The results show that with increasing the ratio of fractal dimensions or apertures of the two fracture sets, the direction of the maximum permeability moves towards the orientation of fractures with a larger fractal dimension or aperture. The ratio of the maximum and the minimum permeability decreases with the increment of intersecting angle of the two fracture sets, following exponential functions. For the ratio of apertures of the two sets of fractures varying from 1 to 5, the ratio of the maximum and the minimum permeability would decrease from 9.93 to 4.55 and increase from 2.79 to 5.35 for intersecting angle having the values of 30 degree and 60 degree, respectively. The larger orientation variance results in the smaller difference between the maximum and the minimum permeability. The reduction/increase rate of permeability decreases and increases for the models with orientation normally and uniformly distributed, respectively.

Keywords: directional permeability; fractal; gas shale; anisotropy; fracture network 


\section{Introduction}

Although permeability of gas shale is controlled by both the fractures and the matrixes of shale, which has attracted much attention during the past several decades, the main pathways for fluid flow are governed by the connected fracture networks, despite its relatively low matrix permeability (Chen et al. 2015a). The fluid flow in gas shale is assumed to obey the linear Darcy's law, in which the flow rate is linearly related with the pressure gradient (Wang et al. 2009; Zhao et al. 2011; Vandenboer et al. 2014; Chen et al. 2015b). The fractures inside of gas shale are commonly invisible, therefore it is a challenging task to accurately describe their geometric properties, such as distributions of length, aperture, orientation, surface roughness, and location (Parashar and Reeves 2012; Wang et al. 2015a, 2015b). Different approaches have been devoted to characterize them, among which the fractal approach has achieved great success in describing the distribution of fracture length and in the analysis of fluid flow and/or solute transport behaviors in the fracture networks (Jafari and Babadagli 2012; Cai et al. 2010, 2013; Miao et al. 2015).

The permeability of fractured rock masses has been studied and/or predicted through various ways, such as in-situ measurement, laboratory experiment, theoretical derivation, and numerical simulation. Bustin et al. (2008) measured the permeability of shales and found that it varies by several orders of magnitude with varying effective stresses. An exponential relationship between shale permeability and effective stress for Muskwa, Barnett and Ohio shale samples was depicted. Zhang et al. (2016) experimentally studied the directional permeability of reservoir rocks based on a specialized permeability apparatus MFORR (multi-functional outburst research rig), and found this apparatus could be utilized as a direct way to analyze the gas displacement process by injecting pure $\mathrm{N}_{2}$ through the drilling hole to the $\mathrm{CH}_{4}$ - or the $\mathrm{CO}_{2}$ - saturated coal. Chen et al. (2015a) derived a theoretical correlation between shale permeability and effective stress, and discussed the relationship of fracture compressibility and shale properties. The results showed that fracture permeability decreases significantly with the decrease of reservoir pressure and the Young's modulus' anisotropic ratio plays an important role in estimating the horizontal fracture permeability drop. Zhu et al. (2016) developed a fully coupled numerical model of a fractured horizontal well to simulate the real gas flow in shale reservoir and to investigate the effect of thermal recovery based on hydraulic fracture heating. The results illustrated that the hydraulic fracture heating could enhance shale gas recovery by altering gas desorption behavior, and is a potentially suitable way for long-term production. The permeability of fracture networks has been confirmed to be directional due to the anisotropic distributions of fracture properties (i.e., length and orientation) (Nur 1971; Leary and Henyey 1985; Schoenberg 1995; Koyama et al. 2006; Barton and Quadros 2014), and the 
directional permeability has been studied in some previous works (Snow 1969; Long et al. 1982; Vu et al. 2013). Oda et al. (1987) established a permeability tensor in terms of two tensors and a nondimensional scalar depending on the geometric aspects (i.e., spacing, orientation, and aperture) of fractures. The prediction of the proposed formulation on permeability tensor agrees well with the results of a large-scale hydraulic conductivity test. Shapiro et al. (1999) proposed an approach for estimating the permeability tensor utilizing seismic emission. The results showed that the rock volume is characterized by a significant permeability anisotropy depending on the oriented crack systems. Baghbanan and Jing (2008) investigated the effects of fracture aperture distribution on the directivity of equivalent permeability, and found that fracture aperture distribution plays a significant role on calculating the directivity of equivalent permeability. Ren et al. (2015) performed numerical simulations using a pipe network method to investigate the permeability anisotropy of 2D fractured rock masses with different fracture distributions. They reported that the permeability anisotropy is mostly affected by the aperture size and distribution, followed by the intersecting angle between two fracture sets, and the number and length ratio in fracture networks. The fracture networks are comprised by hundreds/thousands of fractures with different lengths, apertures, and orientations, and it is difficult to accurately describe them of each fracture (Zhang and Nemcik 2013; Liu et al. 2016a, 2016b; Li et al. 2016). Fortunately, these parameters (i.e., fracture length and aperture) show fractal properties, and the fractal analysis of thermal conductivity (Li et al. 2014; Xu 2015), permeability (Xiao et al. 2012; Liu et al. 2015; Xu et al. 2016) and spontaneous imbibition (Cai et al. 2014, 2015) is believed to be effective and has developed rapidly in the recent years. Jafari and Babadagli (2013) presented that the percolation-fractal properties are closely related with the permeability of 2-D fracture networks. They concluded that the fractal dimension of fracture lines that were obtained utilizing the box counting method yields a more accurate prediction, comparing with those of connectivity index, scanning lines in $X$ - and $Y$-directions, and intersection point. Kruhl (2013) showed a review work that quantifies the complex rock structures including inhomogeneity, anisotropy, and scale effect of rock masses based on the fractal-geometry techniques. Liu et al. (2015) theoretically derived a fractal model in which fracture length distribution follows fractal characters to estimate the equivalent permeability of fracture networks, and discussed the relationship between permeability and fractal dimensions. The results showed that permeability variation is approximately five orders of magnitude when the fractal dimension is small (i.e., 1.3), and gradually reduces to less than two orders of magnitude for larger fractal dimension (i.e., 1.8). Liu et al. (2016c) later derived a new governing equation for fluid flow in fractures and incorporated it into the previously proposed fractal model, and investigated the effects of random numbers for generating fracture location, orientation, and length. They found that 
permeability is more dependent on the random number utilized to generate fracture length than those to generate location and orientation. However, in the previous works, fracture orientation was assumed to be uniformly and randomly distributed in a range of $[0,2 \pi)$, which might deviate from the natural cases in which orientation of fractures commonly follows some other distributions (i.e., lognormal and/or normal distributions). For example, as shown in Fig. 1, the fractures at both Beishan, China, and Kyushu Island, Japan, exhibit strong directivities, and the orientation deviates from its mean values to some extent. Therefore, the anisotropy of fracture distribution should be considered when calculating the permeability of real fracture networks.

In this study, to characterize the directional permeability of gas shale fracture networks, a series of discrete fracture network (DFN) models that are consisted of two fracture sets were established, in which fracture length distribution follows the fractal scaling law. The influences of fractal dimension, aperture, and variance of orientation on the permeability tensor (both magnitude and direction) were systematically investigated. Some empirical expressions were proposed to predict the variations of the ratio of the maximum permeability to the minimum permeability and the orientation of the maximum permeability for different cases.

\section{A new expression for fractal length distribution of fractures}

In the previous works, a fractal length distribution of fractures was proposed, and its validity was successfully verified by comparing theoretical values with in-situ measurements (Liu et al. 2015, 2016c). The fracture length, $l_{i}$, of the $i$ th fracture in a fracture network could be calculated as below:

$$
l_{\mathrm{i}}=\frac{l_{\min }}{\left(1-R_{i}\right)^{2 / D}}=\left(\frac{l_{\min }}{l_{\max }}\right) \frac{l_{\max }}{\left(1-R_{i}\right)^{2 / D}}
$$

where $D$ is the fractal dimension of fracture backbone, $i=1,2,3, \ldots, N_{t}$, and $N_{t}$ is the number of fractures in the fracture networks, $l_{\max }$ are the maximum trace length of fractures, $l_{\min }$ are the minimum trace length of fractures, and $R_{i}$ is the random number between 0 and 1 . The process of deriving Eq. (1) is detailedly described in Liu et al. (2015).

However, the orientation was assumed to be uniformly and randomly distributed by neglecting its anisotropy, which is different from the natural cases. Besides, Fig. 1(a) shows that the number of the fractures for set 1 is 357, and for set 2 is 708; while Fig. 1(b) presents that the number of the fractures for set 2 is much larger than that for set 1 . Therefore, the fractal dimension of the fracture backbone, which is utilized to describe the relationship of fracture number and length, is alternated for different fracture sets. By incorporating a subscript $j$, Eq. (1) gives: 


$$
l_{i j}=\frac{l_{\min }}{\left(1-R_{i j}\right)^{/ D_{j}}}=\left(\frac{l_{\min }}{l_{\max }}\right) \frac{l_{\max }}{\left(1-R_{i}\right)^{2 / D_{j}}}
$$

where $l_{i j}$ is the trace length of the $i$ th fracture for fracture set $j, D_{j}$ is the fractal dimension of fracture backbone consist of fracture set $j, j=1,2,3, \ldots, N_{s}$, and $N_{s}$ is the total number of fracture set. In the present work, only two fracture sets were considered by assigning $N_{s}=2$. Eq. (2) is the new expression for the fractal length distribution of fractures, which incorporates two fracture sets with different fractal dimensions.

\section{DFN generation and boundary conditions}

Assumptions were made that fracture networks were consisted of two fracture sets, and the mean orientation of one fracture set was horizontal ( 0 degree), while the second fracture set has a mean orientation of $\theta_{m}$ that varies from 30 degree to 90 degree. Therefore, $\theta_{m}$ is also the intersecting angle of the two fracture sets. The location of fractures was uniformly and randomly distributed in the range of concerned region, and orientation of fractures followed normal distributions. The mean values of normally distributed orientations for the two fracture sets were 0 degree and $\theta_{m}$, and the variances were both 10 degree. The apertures of both fracture sets ( $e_{1}$ and $\left.e_{2}\right)$ were assumed to be the same constant with a value of $65 \mu \mathrm{m}$, which is the same magnitude with the in-situ measurements (Baghbanan and Jing 2008). $l_{\min }=0.5 \mathrm{~m}$ and $l_{\max }=500 \mathrm{~m}$, with a ratio of 0.001 (Liu et al. 2015). Both fractal dimensions ( $D_{1}$ and $\left.D_{2}\right)$ of the two fractures sets ranged from 1.3 to 1.5 , below which the fracture networks were not well connected and above which there were so many fractures/segments that is beyond the capability of a common computer. Different combinations of $D_{1}$ and $D_{2}$ and the values of other parameters used for DFN generation were tabulated in Table 1. When analyzing one parameter, the others were fixed and assigned initial values. Fig. 2 presents three of the fracture network models with different fractal dimensions, side lengths $(L)$, orientations or intersecting angles $\left(\theta_{m}\right)$ for the two fracture sets. From these figures, we can see that when the fractal dimension is small, the fracture network is composed of relative small fractures (see Figs. 2(a) (c)), and for the fracture network having a large fractal dimension, it is composed of relative large fractures (see Figs. 2(g) (i)). With increasing $D_{2}$ and fixing $D_{1}=1.3$, the mass density of the two fracture sets seems to be different, indicating that the permeability might be anisotropic if assigning a constant aperture to all fractures. The relationship of fractal dimensions $\left(D_{1}\right.$ and $\left.D_{2}\right)$ and permeability tensor (both magnitude and directivity) would be presented in the following Section 4.2. 
To calculate the permeability tensor of a fracture network, the model scale should be equal to or larger than the REV (representative elementary volume) size, below which the permeability tensor might vary significantly and beyond which the permeability tensor would hold almost constant values. To calculate the REV size for the fracture networks with different $D_{1}$ and $D_{2}$, smaller DFNs were extracted from original large models with different rotations $\left(\theta_{r}\right)$ as illustrated in Fig. 3. A pressure gradient was fixed at $10 \mathrm{kPa} / \mathrm{m}$ to generate flow along the rotated direction by applying different pressures on the opposing sides. The other two sides that are perpendicular to the flow direction were assumed to be impermeable. The minimum side length $\left(L_{\min }\right)$ and the maximum side length $\left(L_{\max }\right)$ of extracted models for different $D_{1}$ and $D_{2}$ were shown in Table 1.

The fluid flow was assumed to obey the linear Darcy's law, and when the elevation head is neglected, the governing equation could be expressed as (Bear 1972; Min et al. 2004):

$$
Q_{\mathrm{i}}=A \frac{K_{i j}}{\mu} \frac{\partial P}{\partial x_{j}}
$$

where $Q_{i}$ is the flow rate, $A$ is the cross-sectional area, $K_{i j}$ is the permeability tensor, $\mu$ is the dynamic viscosity, and $P$ is the hydraulic pressure.

A two-dimensional DEM (discrete element method) code UDEC (Itasca, 2004) was developed by the authors to calculate the flow rate of fracture networks generated above. The details of the DEM code were described in the previous works of Liu et al. (2015, 2016c). After the flow rate is obtained, the permeability could be back-calculated according to Eq. (3).

\section{Results and analysis}

\subsection{Modeling verification}

In the previous works of Ren et al. (2015), the variations of PAR (permeability anisotropy ratio) that is defined as the ratio of permeability in the horizontal direction to that in the orthogonal direction were characterized as shown in Fig. 4, based on the fracture network models containing two fracture sets. In their model, the first fracture set was fixed with an inclination angle of 30 degree, and the intersecting angle of the two fracture sets ranged from 20 degree to 140 degree in the counter clockwise direction. To compare the variations of PAR in the present study to those in the works of Ren et al. (2015), PAR should be the ratio of permeability at $\theta_{r}=330$ degree to that at $\theta_{r}=60$ degree, because the first fracture set was fixed with an inclination angle of 0 degree in the present study. Based on the fracture network models as shown in Figs. 2 (a) (c), the values of PAR were calculated and plotted in Fig. 4 with the intersecting angle (represented by $\theta_{m}$ in Fig. 2) ranging from 30 degree to 90 degree. The results show that with the increment of intersecting angle 
from 30 degree to 90 degree, PAR decreases and then increases for both models, although the values of PAR in the present model are slightly less than those in Ren's model. The reasons might be that the variance of orientation for the two fracture sets was the same 10 degree in the present model, whereas it was 0 degree in Ren's model. Therefore, the validity of the proposed fractal length distribution of fractures (see Eq. (2)) and the validity of the proposed DFN modeling technics could be efficiently verified.

\subsection{Effect of fractal dimension}

For each case in Table 1, 8 smaller models were extracted from the original large model, and each smaller model was rotated 12 times with $\theta_{r}=0 \sim 165$ degree, resulting in a total of $96 \times 6=$ 576 fracture network models. The variations of permeability $(K)$ with different $L, D_{1}$ and $D_{2}$ for the case of $\theta_{m}=30$ degree are presented in Fig. 5. The results show that when $L$ is small, $K$ varies significantly due to the influences of random numbers utilized to generate fracture location, orientation, and length. When $L$ exceeds some certain values, $K$ holds constants despite of $\theta_{r}$, in which case the model scale could be regarded as the REV size and the corresponding side length of DFNs is represented by $L_{R E V} . L_{R E V}=15 \mathrm{~m}, 9 \mathrm{~m}$, and $6 \mathrm{~m}$ for the cases in Figs. 5 (a) (c), respectively. The relationship of $L_{R E V}$ and the mean fractal dimension $\left(D_{m}\right)$ of the two fracture sets is depicted in Fig. 6, in which it shows that with the increment of $D_{m}, L_{R E V}$ decreases following an exponential function. This equation could be utilized to predict the REV size of a fracture network that is consisted of two fracture sets.

To calculate the permeability tensor (both magnitude and direction), Fig. 7 shows nine of the directional permeability contours with different fractal dimensions and orientations for the fracture networks at the REV size. The results show that when $D_{1}$ is fixed as 1.3 , with the increment of $D_{2}$, both the maximum permeability $\left(K_{\max }\right)$ and the minimum permeability $\left(K_{\min }\right)$ increase at a certain $\theta_{m}$ (i.e., 30 degree). With the increasing $\theta_{m}$, the directional permeability contour seems to be more isotropic, comparing with those with smaller $\theta_{m}$ (see Figs. 7(a) $\sim(\mathrm{c})$ ). For all the cases as shown in Table 1 , $K_{\max }$ could be predicted with $D_{m}$, following a linear function as shown in Fig. 8, whereas $K_{\min }$ increases exponentially as $D_{m}$ increases. The variations of $K_{\max } / K_{\min }$, which could be utilized to characterize the degree of anisotropy of permeability, exhibit exponential functions with the increment of $\theta_{m}$ as shown in Fig. 9. The larger value of fractal dimension results in the larger number of fractures, and thereafter the stronger conductivity. Fig. 10 illustrated that the directivity/orientation of the maximum permeability $\left(\theta_{\max }\right)$ has quadratic relationships with the ratio of fractal dimensions of the two fracture sets (represented by $D_{2} / D_{1}$ ). With increasing $D_{2} / D_{1}, \theta_{\max }$ 
increases dramatically, and gradually holds constant values that are close to the orientations of the fracture set with larger fractal dimension.

\subsection{Effect of aperture}

Fracture aperture plays an important role in the conductivity of a fracture network, because the flow rate is linearly proportional to the cube of aperture. In this Section, the case of $D_{1}=1.5$ and $D_{2}$ $=1.5$ was selected to characterize the effect of aperture for the fracture network models with $e_{2} / e_{1}=$ $1.0 \sim 5.0, L=3 \mathrm{~m}$, and $\theta_{m}=30 \sim 60$ degree, where $e_{2} / e_{1}$ is the ratio of apertures of the two fracture sets. As a result, a total of 216 fracture network models were calculated. The directional permeability contours are exhibited in Fig. 11, and the results show that with the increment of $e_{2} / e_{1}$, $K_{\max }$ increases. This is because $e_{1}$ is fixed as $65 \mu \mathrm{m}$, and $e_{2}$ increases from $65 \mu \mathrm{m}$ to $325 \mu \mathrm{m}$. Fig. 12 presents the variations of $K_{\max } / K_{\min }$ with increasing $e_{2} / e_{1}$ from 1.0 to 5.0. $K_{\max } / K_{\min }$ decreases from 9.93 to 4.55 and increases from 2.79 to 5.35 for the cases of $\theta_{m}=30$ degree and 60 degree, respectively, both following quadratic functions. The degree of anisotropy of permeability decreases with the small $\theta_{m}$ (i.e., $\theta_{m}=30$ degree), however, it increases with the large $\theta_{m}$ (i.e., $\theta_{m}=60$ degree). The reasons might be that the increasing rate of $K_{\max }$ is less than that of $K_{\min }$ for $\theta_{m}=30$ degree, however, the increasing rate of $K_{\max }$ is larger than that of $K_{\min }$ for $\theta_{m}=60$ degree. Fig. 13 illustrates that $\theta_{\max }$ has power-law relationships with $e_{2} / e_{1}$. With increasing $e_{2} / e_{1}, \theta_{\max }$ increases dramatically, and gradually holds constant values that are close to the orientations of the fracture set with larger aperture.

\subsection{Effect of orientation variance}

The influences of orientation of fractures on the permeability tensor (both magnitude and direction) have attracted much attentions, however, the orientation variance $\left(\theta_{v a r}\right)$ that could be changed according to different numbers of dataset was commonly negligible. In this Section, $\theta_{\text {var }}$ ranged from 10 degree to 180 degree for both fracture sets, the orientation of which was normally or uniformly distributed. The fracture network models with varying distributions of orientations and corresponding variances are shown in Fig. 14. These models are consisted of fractures with $D_{1}=$ 1.5, $D_{2}=1.5, L=3 \mathrm{~m}$, and $\theta_{m}=30$ degree. With the increment of $\theta_{v a r}$, the fractures are more randomly distributed, and the intersecting angle of the two fracture sets is more deviated from the original 30 degree. Fig. 15 presents the directional permeability contours, and it shows that with the increment of $\theta_{\text {var }}, K_{\max }$ decreases and $K_{\min }$ increases when the fractures are normally distributed. However, when the fractures are uniformly distributed, $K_{\min }$ increases and $K_{\max }$ varies negligibly small. For both cases, we could see that the difference of $K_{\max }$ and $K_{\min }$ would be smaller for larger 
$\theta_{\text {var }}$ as shown in Fig. 16. However, the variation patterns of permeability of the models with orientation normally distributed and uniformly distributed are very different. The reduction/increase rate of permeability decreases for the models with orientation normally distributed, however, the reduction/increase rate of permeability increases for the models with orientation uniformly distributed. Fig. 17 illustrates the influence of $\theta_{\text {var }}$ on $K_{\max } / K_{\min }$, and the results show that the value of $K_{\max } / K_{\min }$ for the models with orientation normally distributed is smaller than that for the models with orientation uniformly distributed, except for the case with $\theta_{v a r}=180$ degree. The value of $K_{\max } / K_{\min }$ reduces dramatically when $\theta_{\text {var }}$ is small, and gradually holds constants for $\theta_{\text {var }}>90$ degree for the models with orientation normally distributed. When orientation of fractures is uniformly distributed, the value of $K_{\max } / K_{\min }$ would be constantly reduced until the end case with $\theta_{v a r}=180$ degree. Therefore, when predicting the permeability tensor of an engineering site, both the distribution of orientations and corresponding variances should be considered.

\section{Conclusions}

A new expression for fractal length distribution of fractures that considers the orientation distribution of fractures was proposed, and a systematic numerical simulation was performed to characterize the directional permeability based on a series of fracture network models. The influences of fractal dimension, aperture, and variance of orientation on the permeability tensor (both magnitude and direction) were investigated.

The results show that with the increment of the mean fractal dimension of the two fracture sets, the side length of DFNs at the REV size decreases following an exponential function, which could be utilized to predict the REV size of a fracture network by calculating its fractal dimensions. The ratio of the maximum permeability to the minimum permeability, which is a factor for characterizing the degree of anisotropy of permeability, exhibits exponential functions with the increment of intersecting angle of the two fracture sets. With the increment of the ratio of fractal dimensions (or apertures) of the two fracture sets, the directivity/orientation of the maximum permeability increases dramatically, and gradually holds constant values that are close to the orientations of the fracture set with larger fractal dimension (or aperture). The larger orientation variance results in the smaller difference between the maximum and the minimum permeability. The reduction/increase rate of permeability decreases and increases for the models with orientation normally and uniformly distributed, respectively. 
This study is focused on the directional permeability of fracture network models containing two fracture sets, and in the future works, fracture network models containing variable (more than two) fracture sets would be incorporated.

\section{Acknowledgments}

This study has been partially funded by National Basic Research 973 Program of China (Grant No. 2013CB036003) and National Natural Science Foundation of China (Grant No. 51379117, 51579239). These supports are gratefully acknowledged.

\section{References}

Baghbanan A, Jing L. Stress effects on permeability in a fractured rock mass with correlated fracture length and aperture. Int J Rock Mech Min Sci 2008, 45:1320-1334.

Barton N, Quadros E. Anisotropy is Everywhere, to See, to Measure, and to Model. Rock Mechanics and Rock Engineering, 2014: 1-17.

Bear J. Dynamics of fluids in porous media, Elsevier, New York, 1972.

Bustin R M, Bustin A M M, Cui A, et al. Impact of shale properties on pore structure and storage characteristics. In: SPE shale gas production conference. Fort Worth, Texas, USA; 16-18 November 2008.

Cai J, Perfect E, Cheng C L, Hu X. Generalized modeling of spontaneous imbibition based on Hagen-Poiseuille flow in tortuous capillaries with variably shaped apertures. Langmuir, 2014, 30(18): 5142-5151.

Cai J, Sun S. Fractal analysis of fracture increasing spontaneous imbibition in porous media with gas-saturated. International Journal of Modern Physics C, 2013, 24(08): 1350056.

Cai J, Yu B, Zou M, et al. Fractal characterization of spontaneous co-current imbibition in porous media. Energy \& Fuels, 2010, 24(3): 1860-1867.

Cai J, Luo L, Ye R, Zeng X, Hu X. Recent advances on fractal modeling of permeability for fibrous porous media. Fractals, 2015, 23(01), 1540006.

Chen D, Pan Z, Ye Z. Dependence of gas shale fracture permeability on effective stress and reservoir pressure: model match and insights. Fuel, 2015a, 139: 383-392.

Chen YF, Hu SH, Hu R, Zhou CB. Estimating hydraulic conductivity of fractured rocks from high-pressure packer tests with an Izbash’s law based empirical model. Water resources research, 2015b, 51(4): 2096-2118.

Itasca Consulting Group Inc. UDEC User’s guide, ver 4.0, Minneapolis, Minnesota, 2004.

Jafari A, Babadagli T. Estimation of equivalent fracture network permeability using fractal and statistical network properties. Journal of Petroleum Science and Engineering, 2012, 92: 110-123.

Jafari A, Babadagli T. Relationship between percolation-fractal properties and permeability of 2-D fracture networks. International Journal of Rock Mechanics and Mining Sciences, 2013, 60: 353-362.

Koyama T, Fardin N, Jing L, Stephansson O. Numerical simulation of shear-induced flow anisotropy and scaledependent aperture and transmissivity evolution of rock fracture replicas. International journal of rock mechanics 
and mining sciences, 2006, 43(1): 89-106.

Kruhl J H. Fractal-geometry techniques in the quantification of complex rock structures: A special view on scaling regimes, inhomogeneity and anisotropy. Journal of Structural Geology, 2013, 46: 2-21.

Leary P C, Henyey T L. Anisotropy and fracture zones about a geothermal well from P-wave velocity profiles. Geophysics, 1985, 50(1): 25-36.

Li L, Yu B, Liang M, Yang S, Zou M. A comprehensive study of the effective thermal conductivity of living biological tissue with randomly distributed vascular trees. International Journal of Heat and Mass Transfer, 2014, 72: 616621.

Li B, Liu R, Jiang Y. Influences of hydraulic gradient, surface roughness, intersecting angle, and scale effect on nonlinear flow behavior at single fracture intersections. Journal of Hydrology, 2016, 538: 440-453.

Liu R, Jiang Y, Li B, Wang X. A fractal model for charactering fluid flow in fractured rock masses on randomly distributed rock fracture networks. Computers and Geotechnics, 2015, 65: 45-55.

Liu R, Jiang Y, Li B. Effects of intersection and dead-end of fractures on nonlinear flow and particle transport in rock fracture networks. Geosciences Journal, doi:10.1007/s12303-015-0057-7, 2016b.

Liu R, Li B, Jiang Y. A fractal model based on a new governing equation of fluid flow in fractures for characterizing hydraulic properties of rock fracture networks. Computers and Geotechnics, 2016c, 75: 57-68.

Liu R, Li B, Jiang Y. Critical hydraulic gradient for nonlinear flow through rock fracture networks: The roles of aperture, surface roughness, and number of intersections. Advances in Water Resources, 2016a, 88: 53-65.

Long JCS, Remer JS, Wilson CR, Witherspoon PA. Porous media equivalents for networks of discontinuous fractures. Water Resour Res 1982;18(3):645-58.

Miao T, Yu B, Duan Y, Fang Q. A fractal analysis of permeability for fractured rocks. International Journal of Heat and Mass Transfer, 2015, 81: 75-80.

Min K B, Jing L, Stephansson O. Determining the equivalent permeability tensor for fractured rock masses using a stochastic REV approach: method and application to the field data from Sellafield, UK. Hydrogeology Journal, 2004, 12(5): 497-510.

Nur A. Effects of stress on velocity anisotropy in rocks with cracks. Journal of Geophysical Research, 1971, 76(8): 2022-2034.

Oda M, Hatsuyama Y, Ohnishi Y. Numerical experiments on permeability tensor and its application to jointed granite at Stripa mine, Sweden. Journal of Geophysical Research: Solid Earth (1978-2012), 1987, 92(B8): 8037-8048.

Parashar R, Reeves D M. On iterative techniques for computing flow in large two-dimensional discrete fracture networks. Journal of computational and applied mathematics, 2012, 236(18): 4712-4724.

Ren F, Ma G, Fu G, Zheng K. Investigation of the permeability anisotropy of 2D fractured rock masses. Engineering Geology, 2015, 196: 171-182.

Schoenberg M, Sayers C M. Seismic anisotropy of fractured rock. Geophysics, 1995, 60(1): 204-211.

Shapiro S A, Audigane P, Royer J J. Large-scale in situ permeability tensor of rocks from induced microseismicity. Geophysical Journal International, 1999, 137(1): 207-213.

Snow D T. Anisotropie permeability of fractured media. Water Resources Research, 1969, 5(6): 1273-1289.

Vandenboer K, van Beek V, Bezuijen A. 3D finite element method (FEM) simulation of groundwater flow during backward erosion piping. Frontiers of Structural and Civil Engineering, 2014, 8(2): 160-166.

Vu M N, Pouya A, Seyedi D M. Modelling of steady - state fluid flow in 3D fractured isotropic porous media: 
application to effective permeability calculation. International Journal for Numerical and Analytical Methods in Geomechanics, 2013, 37(14): 2257-2277.

Wang F P, Reed R M. Pore networks and fluid flow in gas shales. In: SPE annual technical conference and exhibition. New Orleans, Louisiana, USA; 4-7 October 2009.

Wang Z, Li S, Qiao L. Analysis of hydro-mechanical behavior of a granite rock mass for a pilot underground crude oil storage facility in China. Rock Mechanics and Rock Engineering, 2015a, 48: 2459-2472.

Wang Z, Li S, Qiao L. Finite element analysis of hydro-mechanical behavior of an underground crude oil storage facility in granite subject to cyclic loading during operation. International Journal of Rock Mechanics and Ming Sciences, 2015b, 73: 70-81.

Xiao B, Fan J, Ding F. Prediction of relative permeability of unsaturated porous media based on fractal theory and Monte Carlo simulation. Energy \& Fuels, 2012, 26(11): 6971-6978.

Xu P, Li C, Qiu S, Sasmito AP. A fractal network model for fractured porous media. Fractals, Doi:10.1142/S0218348X16500183, 2016.

Xu P. A discussion on fractal models for transport physics of porous media. Fractals, 2015, 23(3): 1530001.

Zhang L, Zhang C, Tu S, Tu H, Wang C. A Study of Directional Permeability and Gas Injection to Flush Coal Seam Gas Testing Apparatus and Method. Transport in Porous Media, 2015: 1-17.

Zhang Z, Nemcik J. Fluid flow regimes and nonlinear flow characteristics in deformable rock fractures. Journal of hydrology, 2013, 477: 129-151.

Zhao Z, Jing L, Neretnieks I, Moreno L. Numerical modeling of stress effects on solute transport in fractured rocks. Computers and geotechnics, 2011, 38(2): 113-126.

Zhu G, Yao J, Sun H, et al. The numerical simulation of thermal recovery based on hydraulic fracture heating technology in shale gas reservoir. Journal of Natural Gas Science and Engineering, 2016, 28: 305-316. 


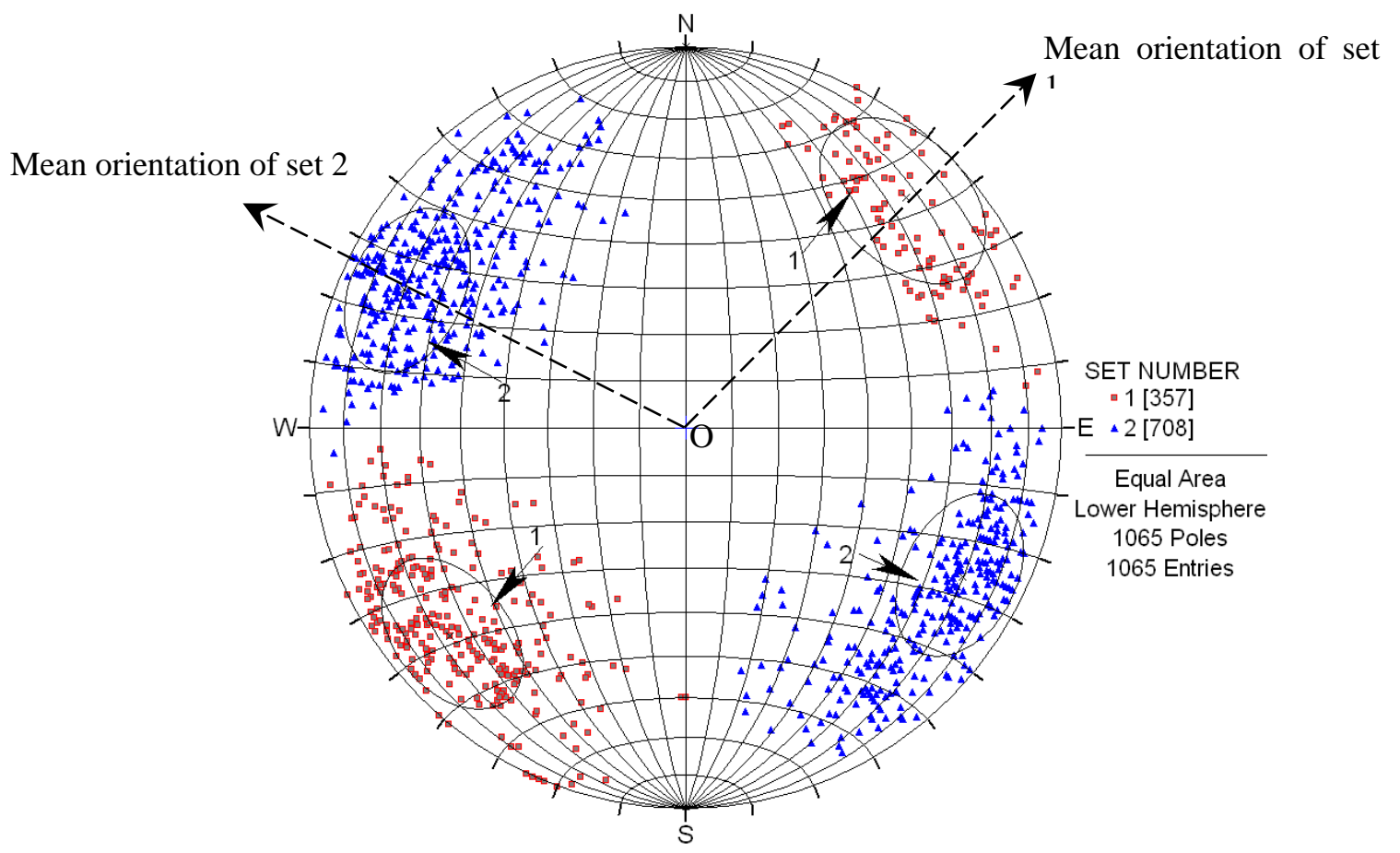

(a) Orientation distribution of fractures at Beishan, China

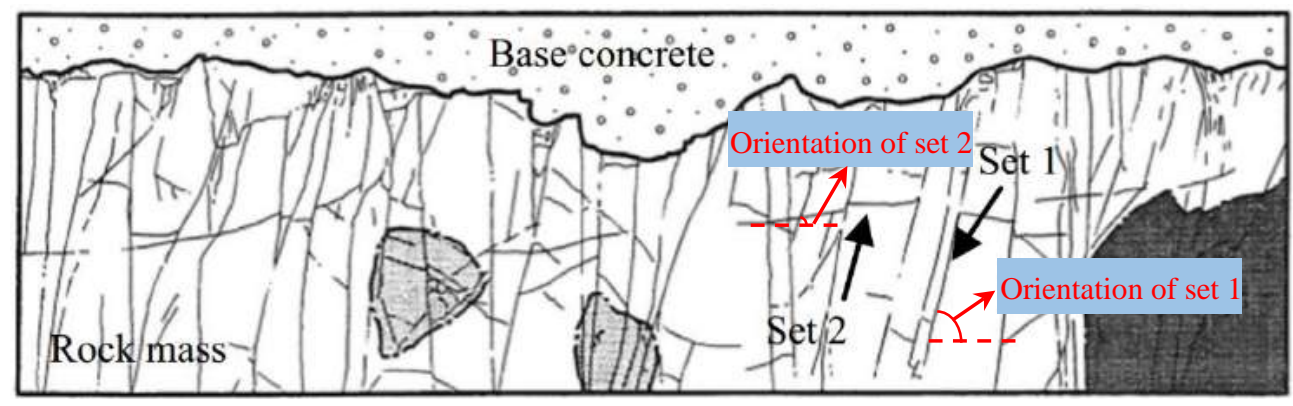

(b) Orientation distribution of fractures at Kyushu Island, Japan

Fig. 1 Orientation distribution of fractures at (a) Beishan, China (after Guo et al. 2015), and (b) Kyushu Island, Japan (after Li et al. 2014). 

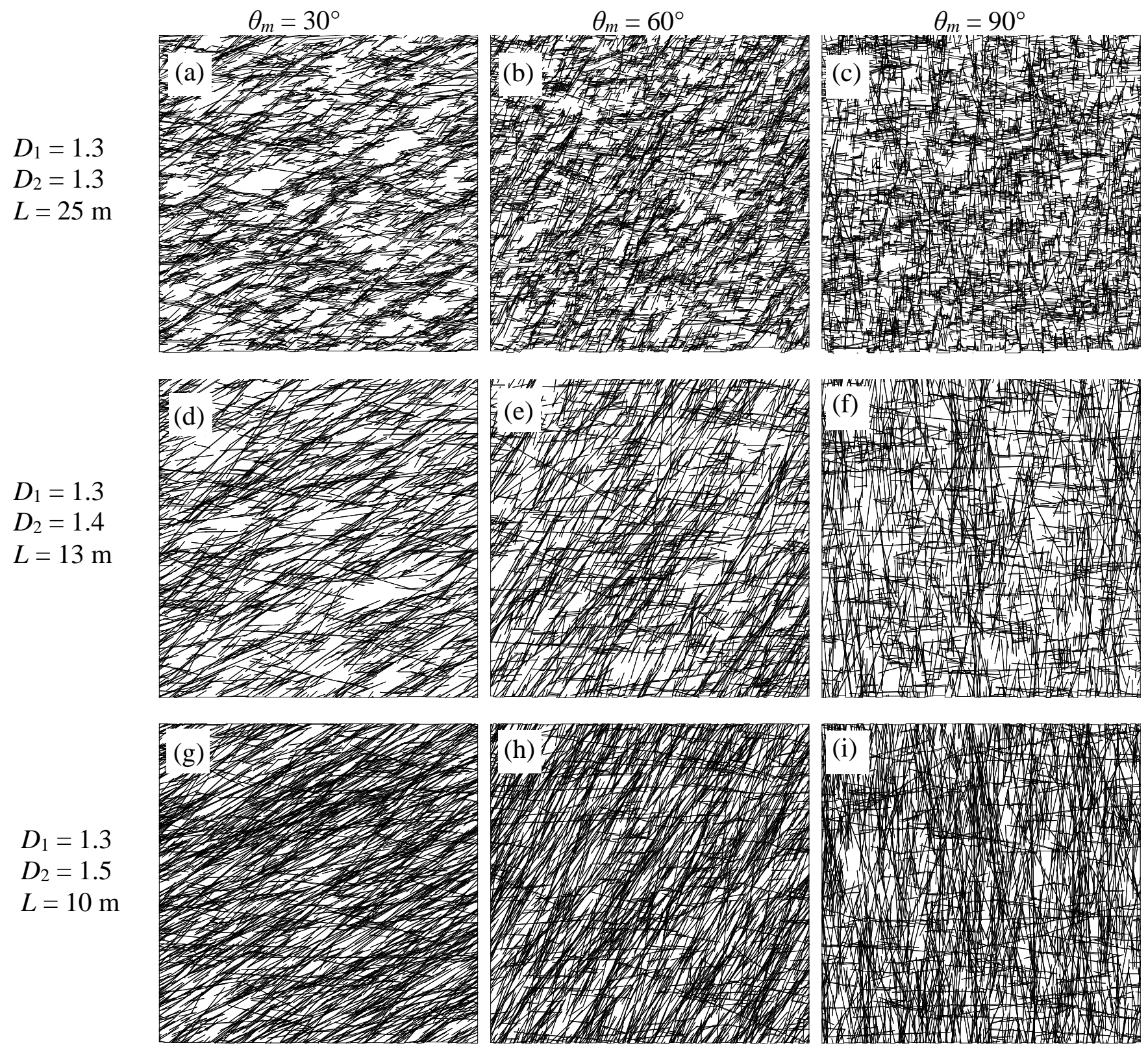

Fig. 2 Fracture network models with different fractal dimensions, side lengths, and orientations for the two fracture sets. 


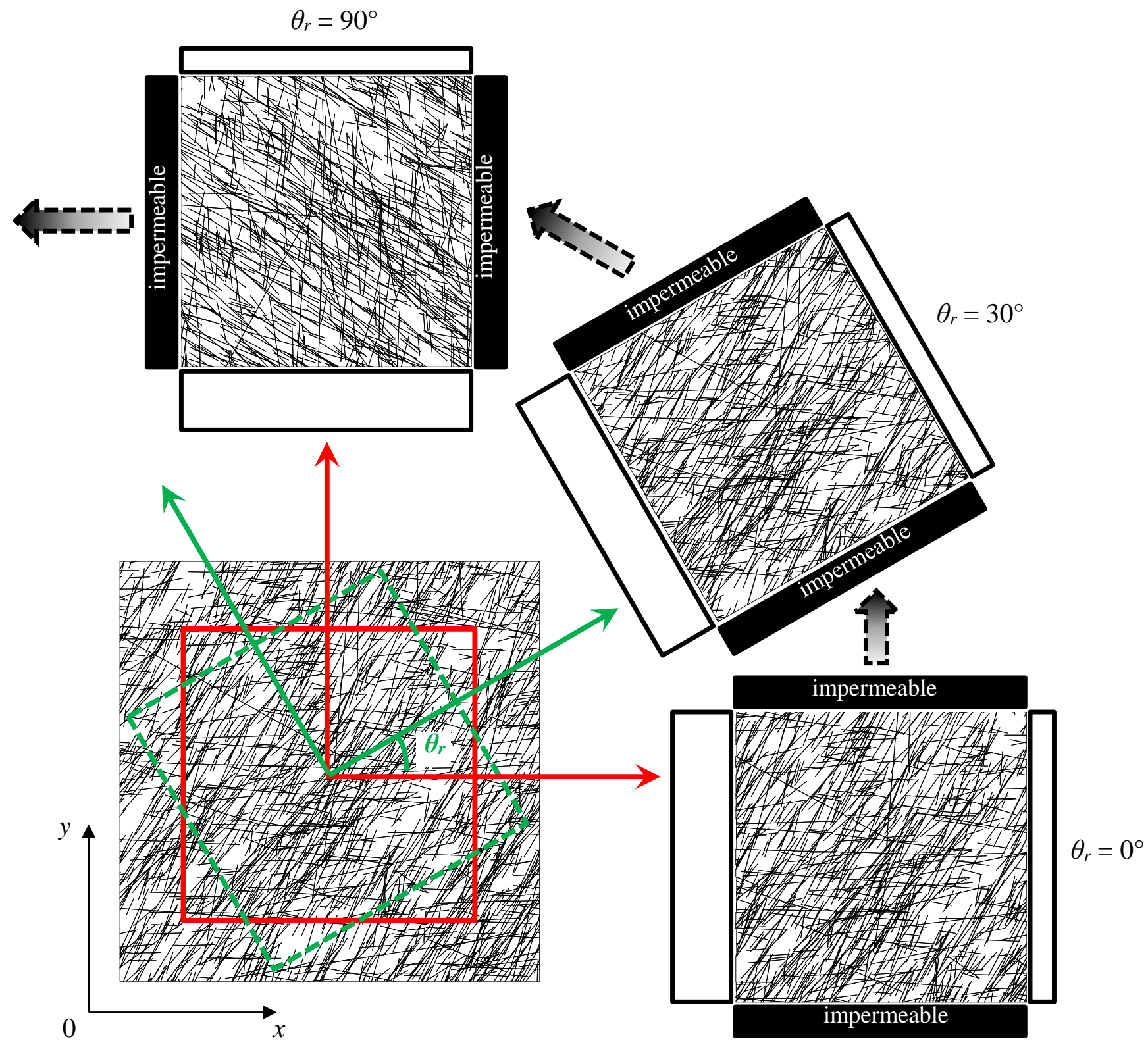

Fig. 3 Extraction of rotated fracture networks from an original large model and corresponding boundary conditions. The quadrilaterals represent hydraulic pressures and the pressure gradient was fixed at $10 \mathrm{kPa} / \mathrm{m}$ to generate flow along the rotated direction. 


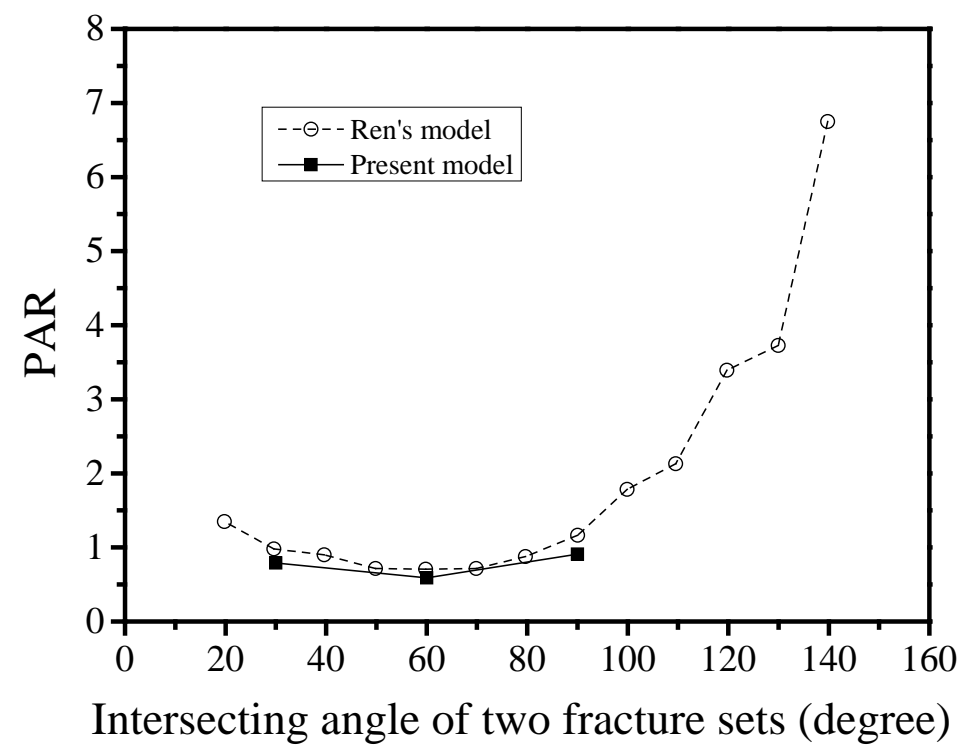

Fig. 4 Comparisons of PAR between Ren's model (after Ren et al. 2015) and the present model. 


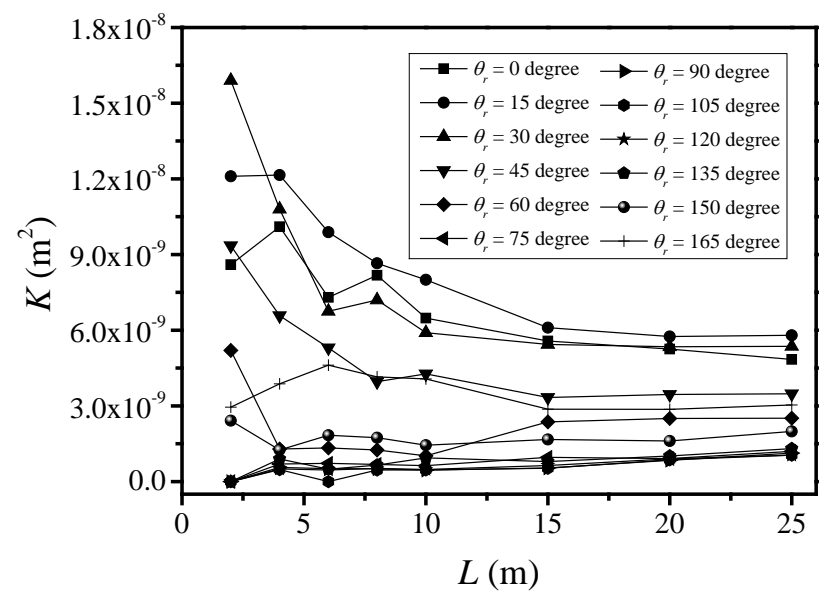

(a) $D_{1}=1.3, D_{2}=1.3$

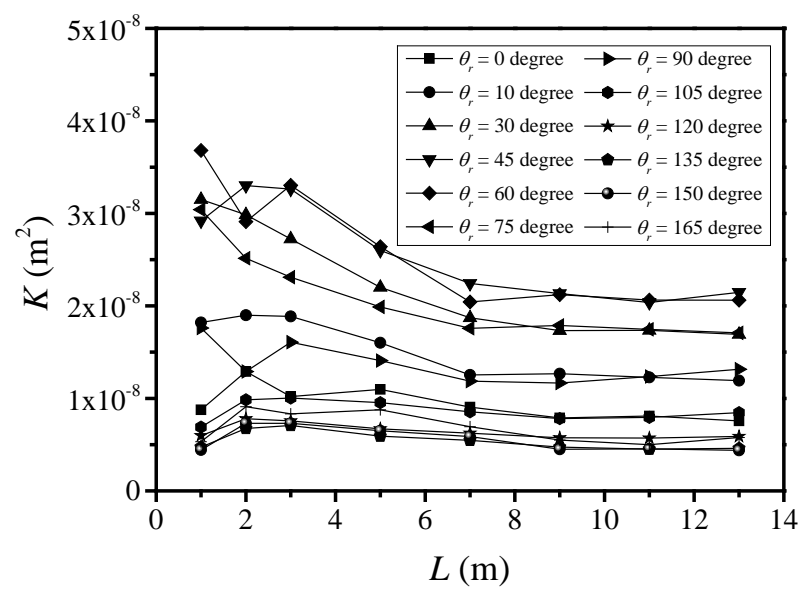

(b) $D_{1}=1.3, D_{2}=1.4$

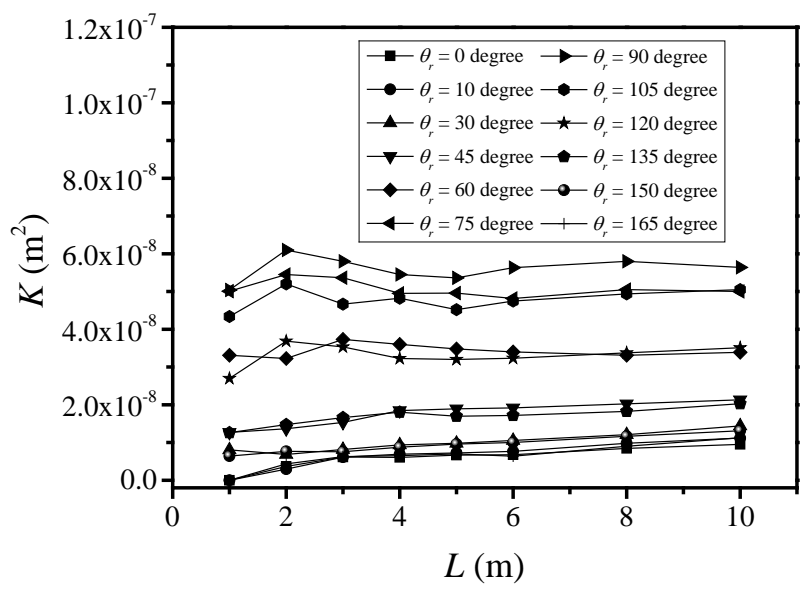

(c) $D_{1}=1.3, D_{2}=1.5$

Fig. 5 Variation of permeability with different DFN side lengths and fractal dimensions. 


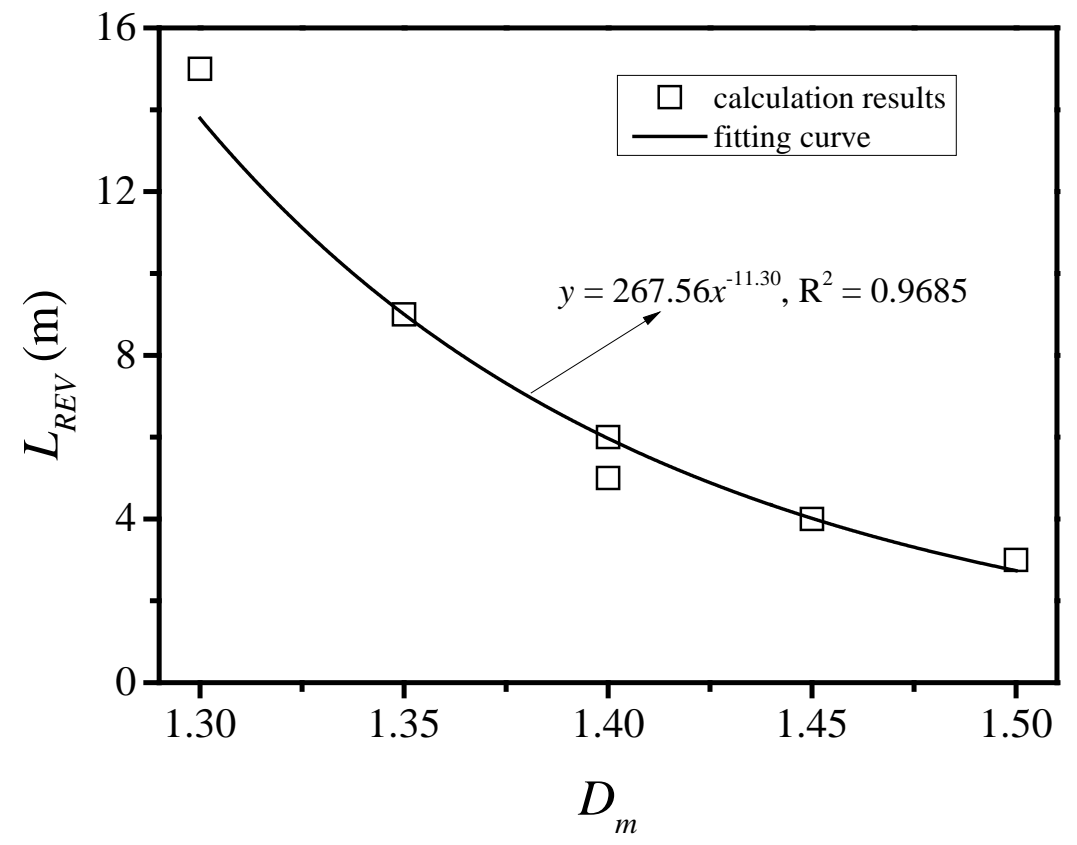

Fig. 6 Relationship between length of fracture networks at the REV size and the mean fractal dimension of the two fracture sets. 


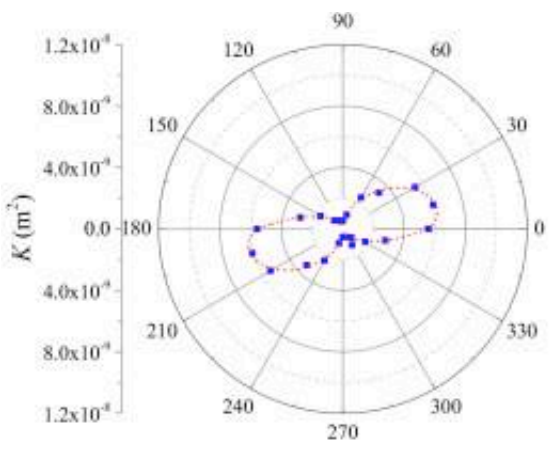

(a) $D_{1}=1.3, D_{2}=1.3, \theta_{m}=30^{\circ}$

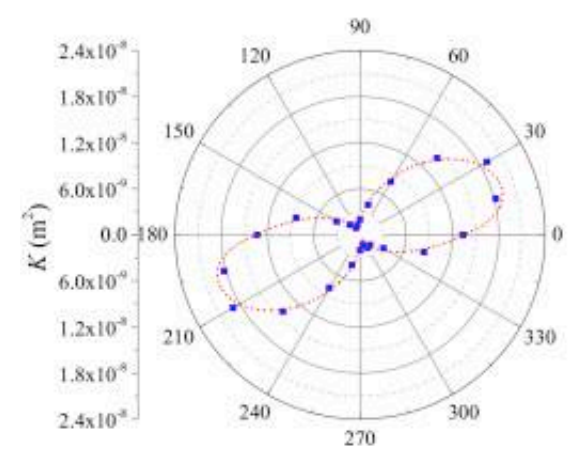

(d) $D_{1}=1.3, D_{2}=1.4, \theta_{m}=30^{\circ}$

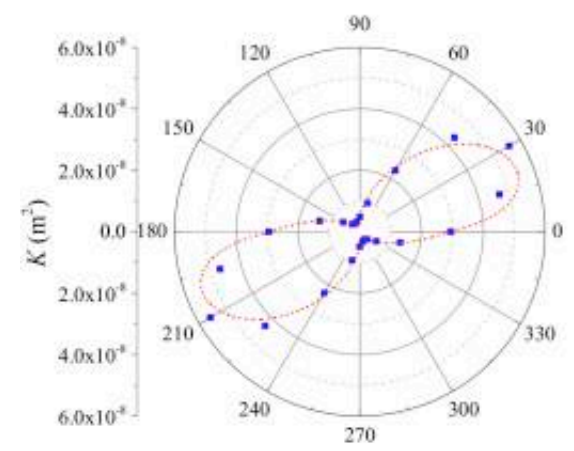

(g) $D_{1}=1.3, D_{2}=1.5, \theta_{m}=30^{\circ}$

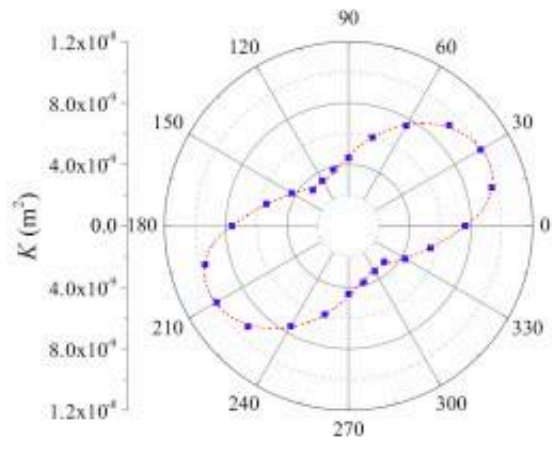

(b) $D_{1}=1.3, D_{2}=1.3, \theta_{m}=60^{\circ}$

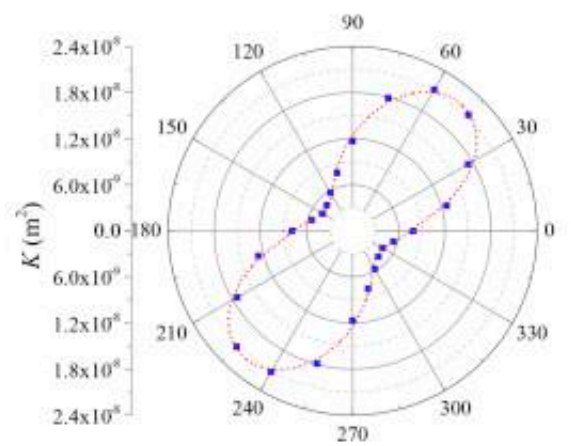

(e) $D_{1}=1.3, D_{2}=1.4, \theta_{m}=60^{\circ}$

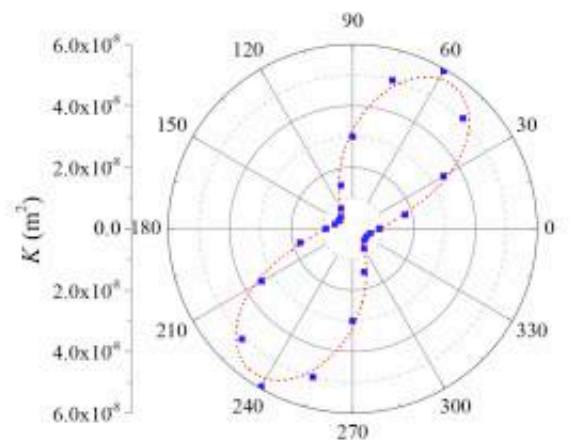

(h) $D_{1}=1.3, D_{2}=1.5, \theta_{m}=60^{\circ}$

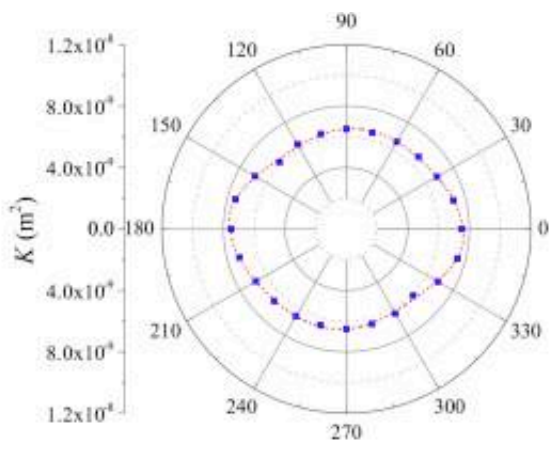

(c) $D_{1}=1.3, D_{2}=1.3, \theta_{m}=90^{\circ}$

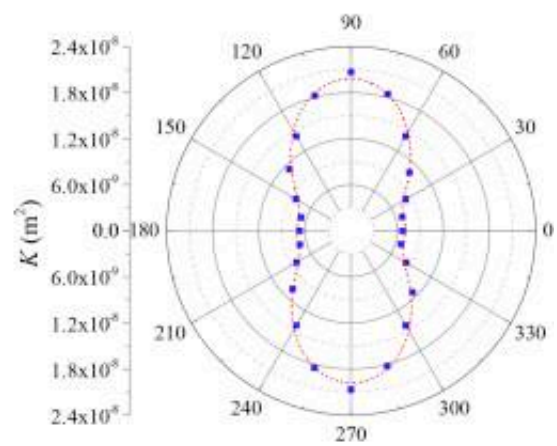

(f) $D_{1}=1.3, D_{2}=1.4, \theta_{m}=90^{\circ}$

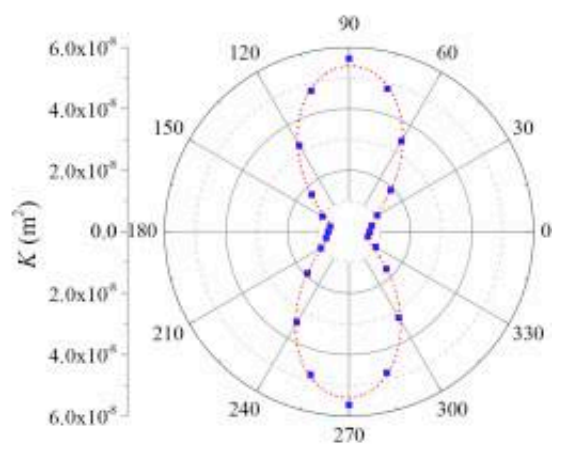

(i) $D_{1}=1.3, D_{2}=1.5, \theta_{m}=90^{\circ}$

Fig. 7 Directional permeability contours with varying fractal dimensions and orientations for the fracture networks at the REV size of $15 \mathrm{~m}$ (a, b, c), $9 \mathrm{~m}$ (d, e, f), and $6 \mathrm{~m}$ (g, h, i). 


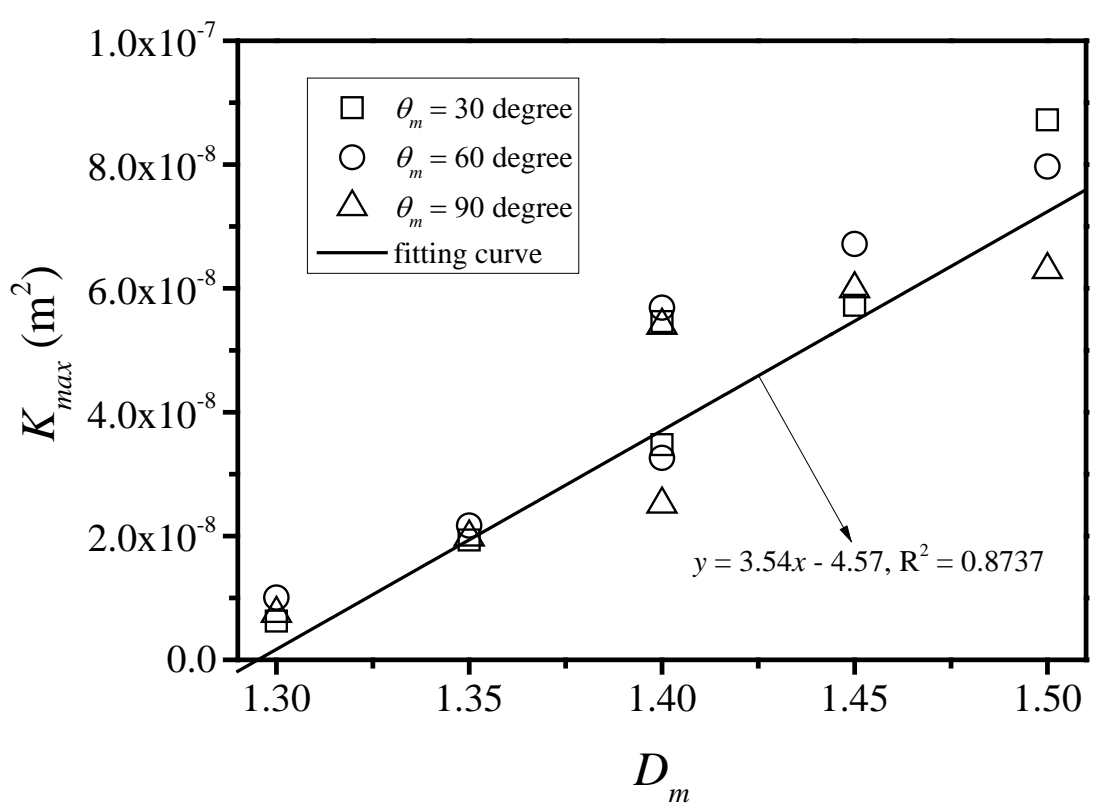

(a) Relationship of $K_{\max }$ and $\left(D_{1}+D_{2}\right) / 2$

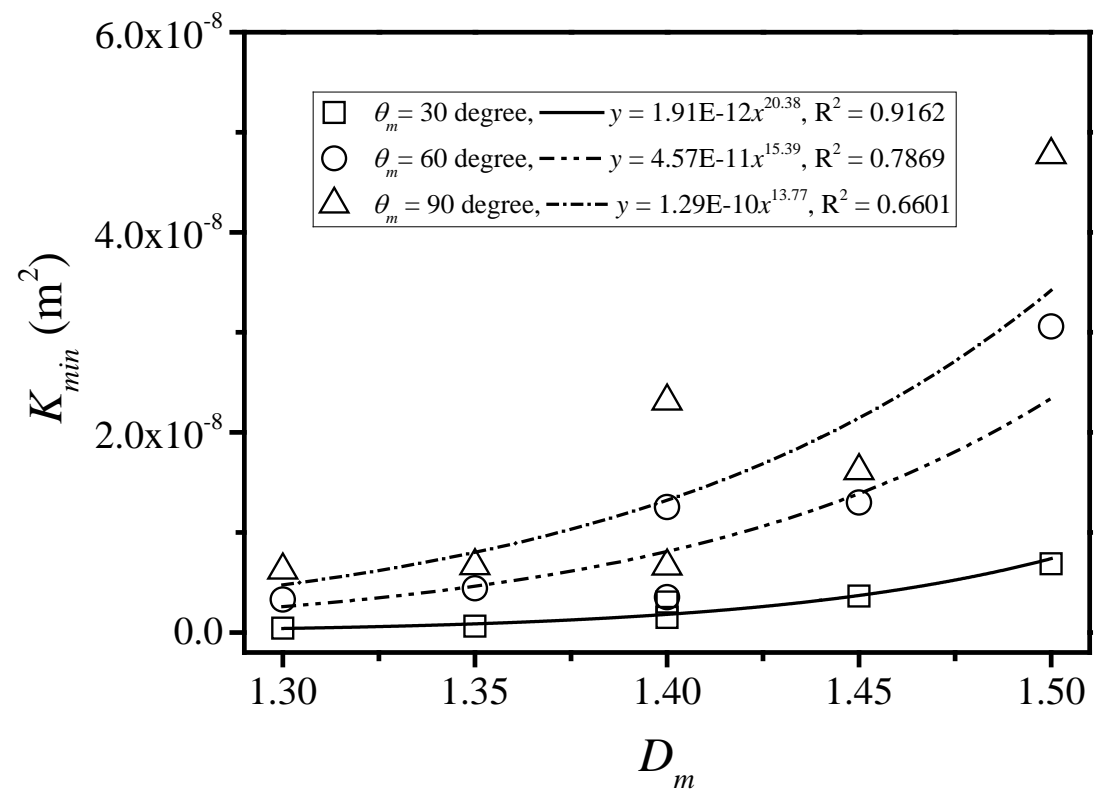

(b) Relationship of $K_{\min }$ and $\left(D_{1}+D_{2}\right) / 2$

Fig. 8 Relationship between the maximum/minimum permeability and the mean fractal dimension of the two fracture sets. 


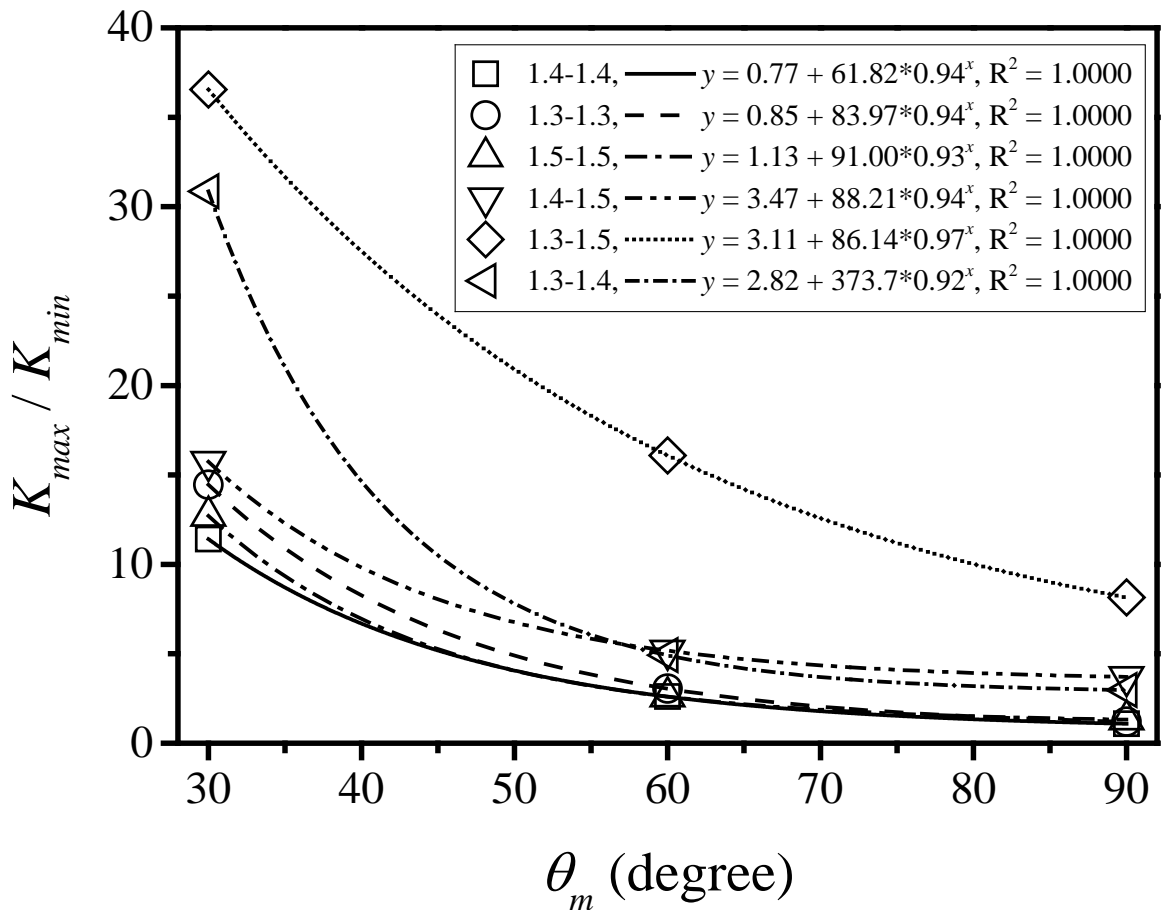

Fig. 9 Variation of the ratio of the maximum permeability to the minimum permeability with increasing orientation from $30^{\circ}$ to $90^{\circ}$. 


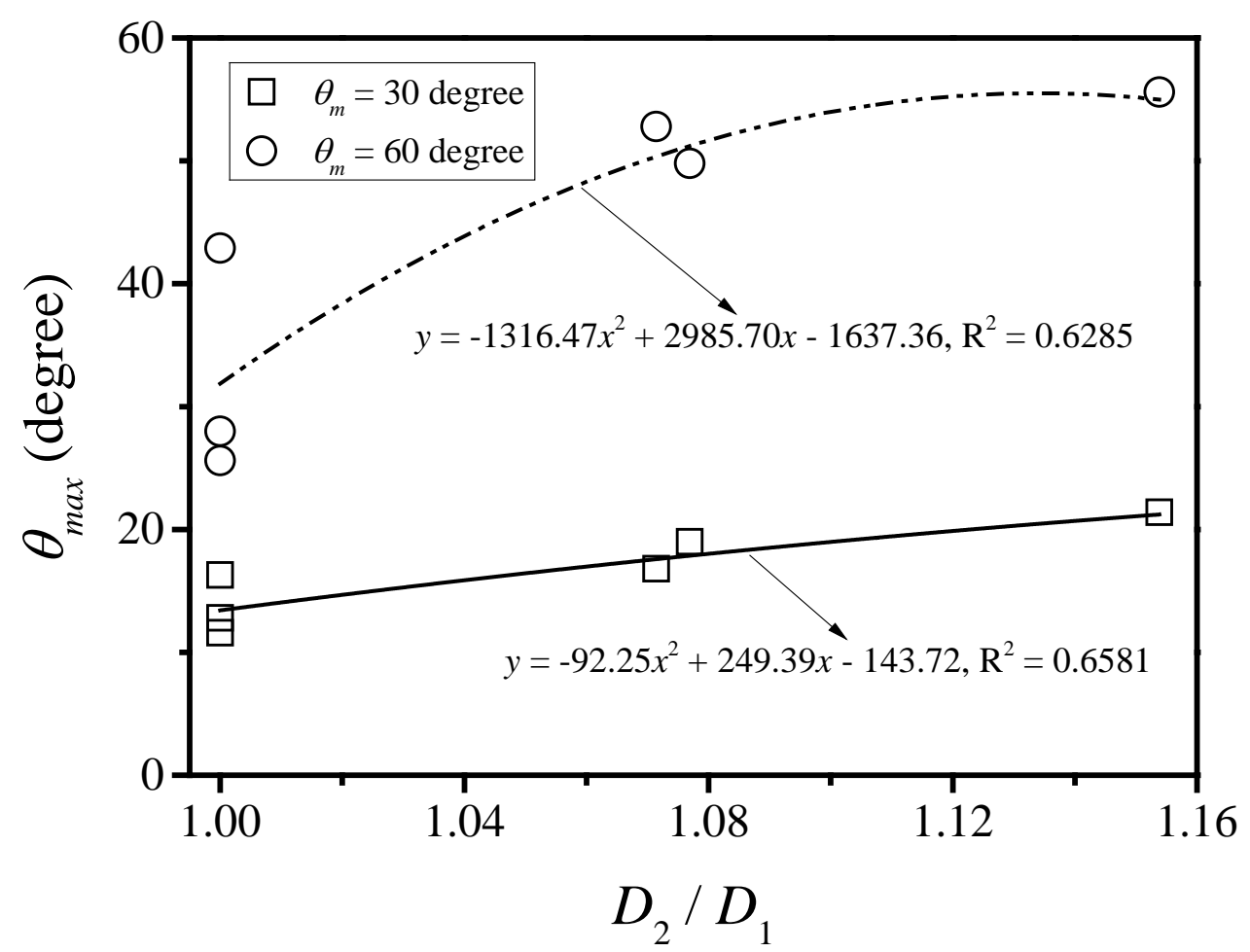

Fig. 10 Orientation of the maximum permeability with ranging the ratio of fractal dimensions of the two fracture sets from 1.00 to 1.15. 


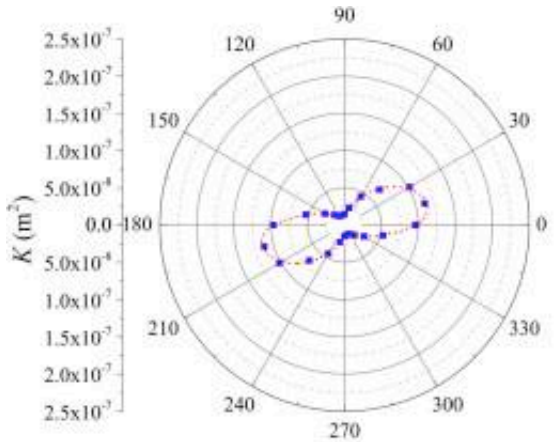

(a) $e_{2} / e_{1}=1.2, \theta_{m}=30^{\circ}$

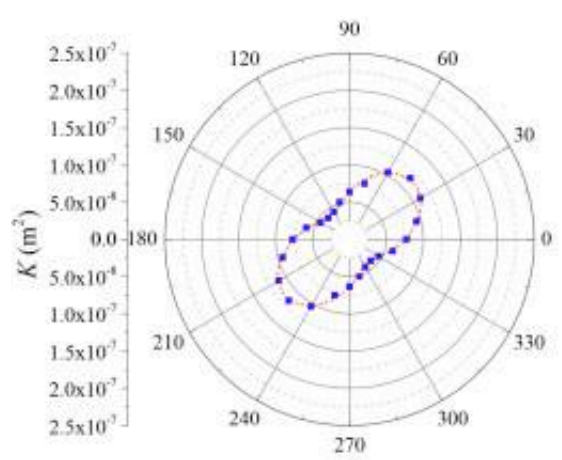

(d) $e_{2} / e_{1}=1.2, \theta_{m}=60^{\circ}$

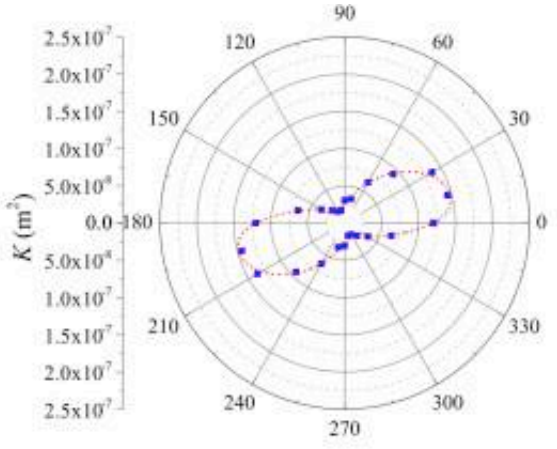

(b) $e_{2} / e_{1}=1.4, \theta_{m}=30^{\circ}$

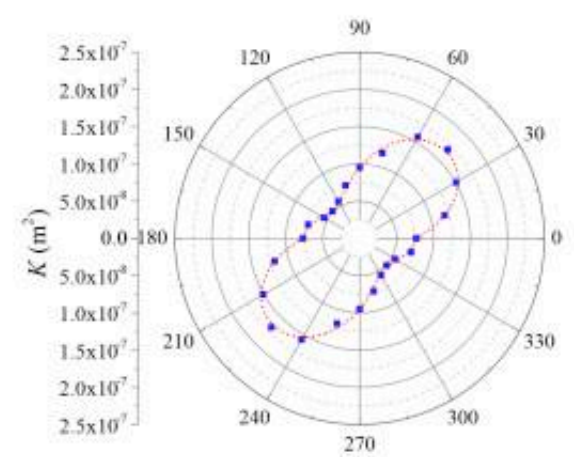

(e) $e_{2} / e_{1}=1.4, \theta_{m}=60^{\circ}$

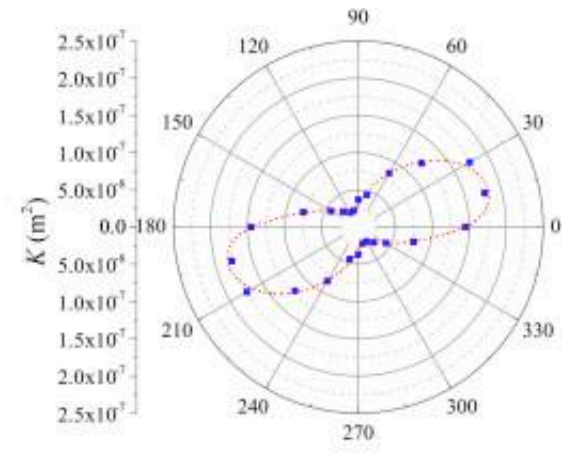

(c) $e_{2} / e_{1}=1.6, \theta_{m}=30^{\circ}$

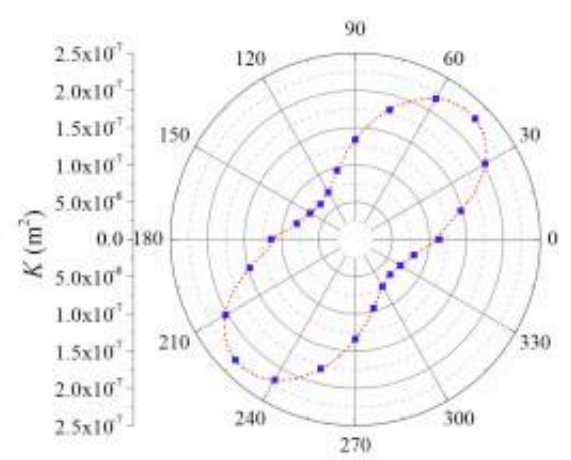

(f) $e_{2} / e_{1}=1.6, \theta_{m}=60^{\circ}$

Fig. 11 Directional permeability contours with varying orientations and varying ratios of the apertures of the two fracture sets. 


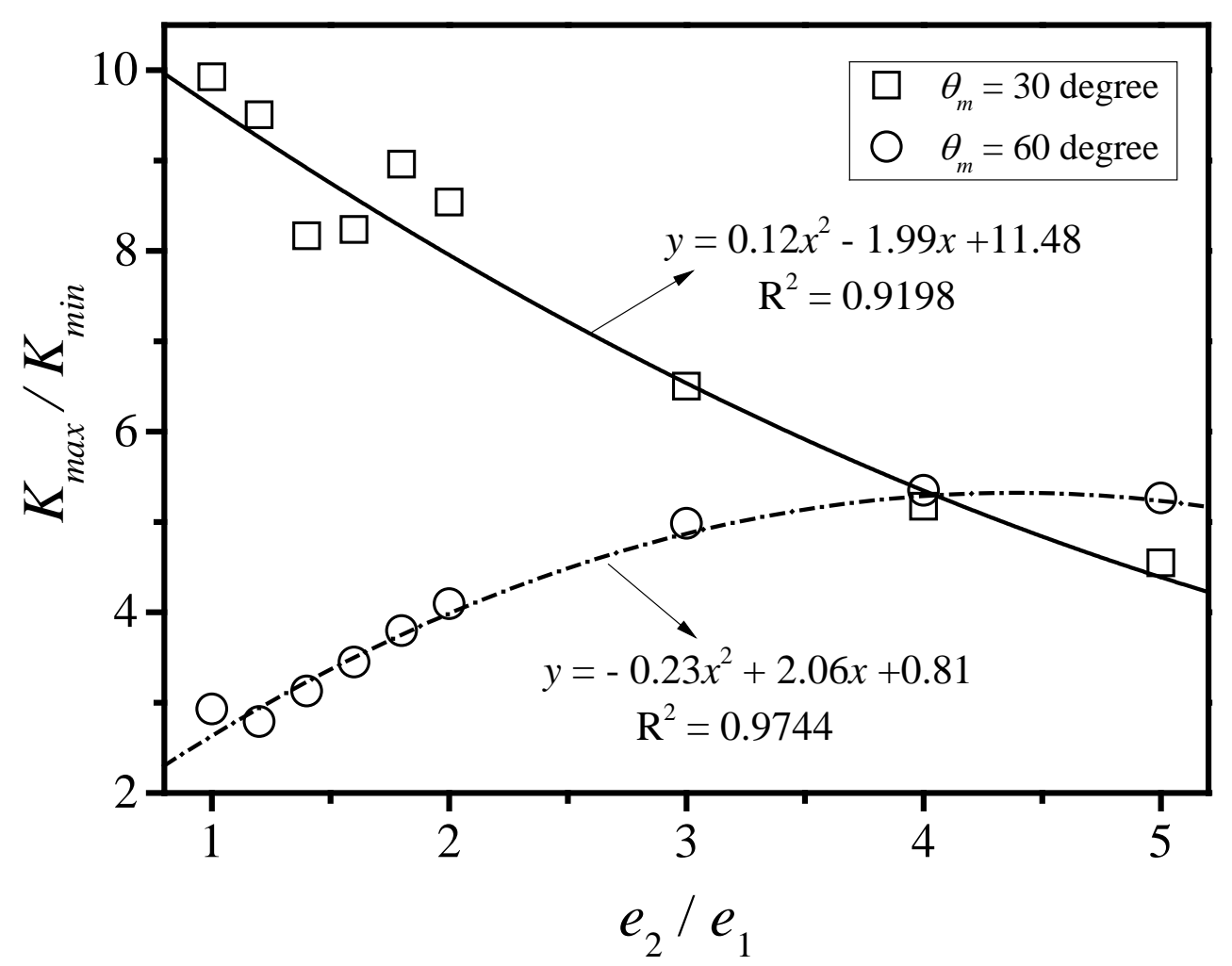

Fig. 12 Relationship between the ratio of the maximum permeability to the minimum permeability and the ratio of the apertures of the two fracture sets from 1.0 to 5.0. 


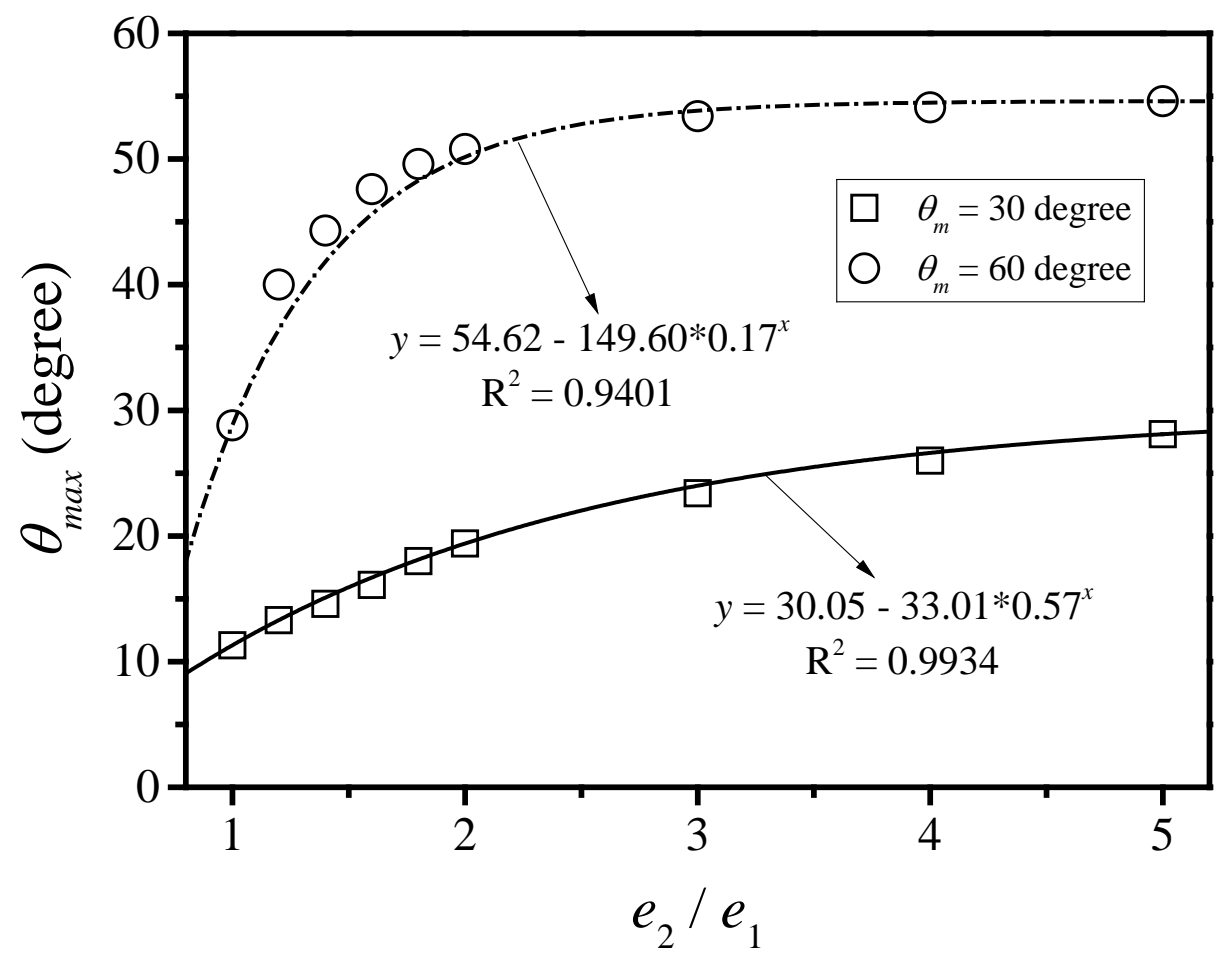

Fig. 13 Orientation of the maximum permeability with ranging the ratio of apertures of the two fracture sets from 1.0 to 5.0 . 


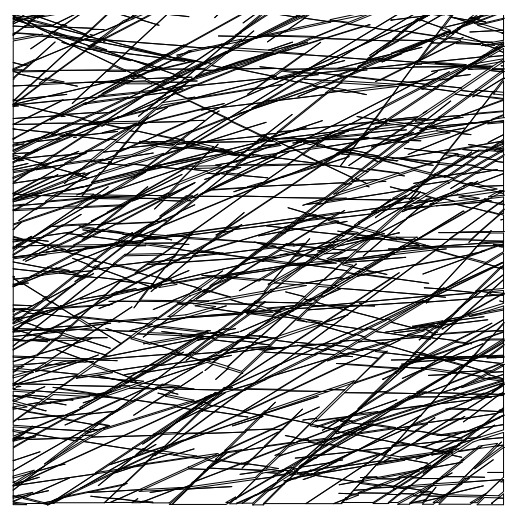

(a) normal distribution, $\theta_{\text {var }}=10^{\circ}$

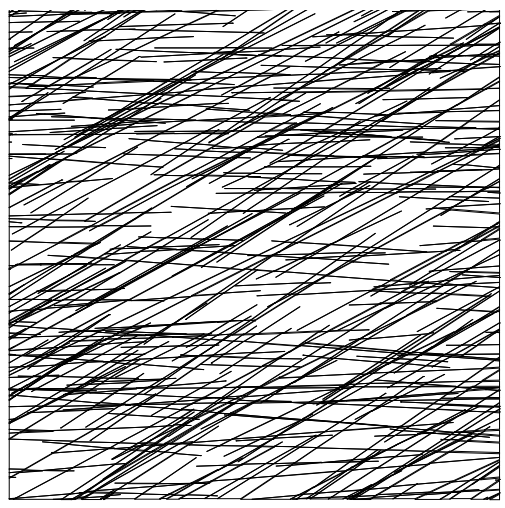

(d) uniform distribution, $\theta_{\text {var }}=10^{\circ}$

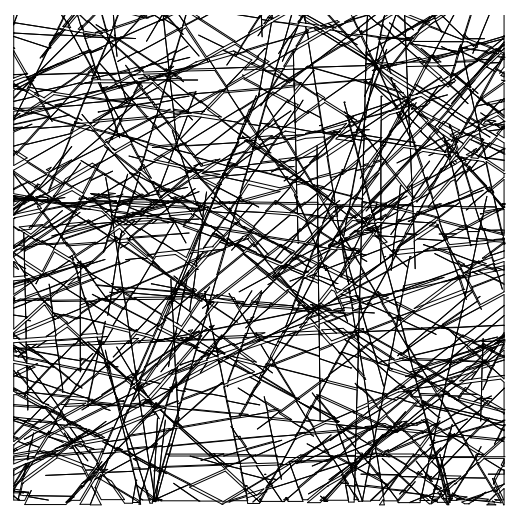

(b) normal distribution, $\theta_{v a r}=50^{\circ}$

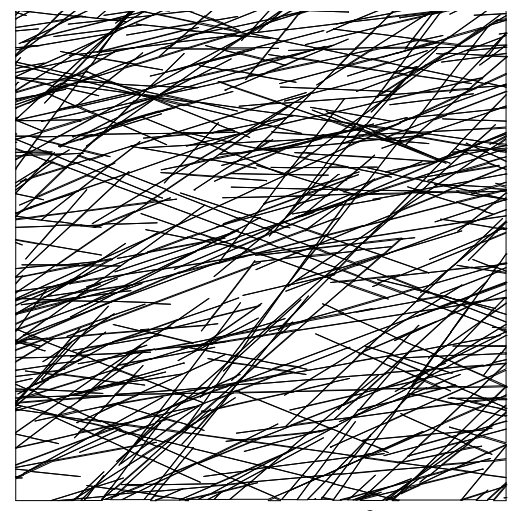

(e) uniform distribution, $\theta_{\text {var }}=50^{\circ}$

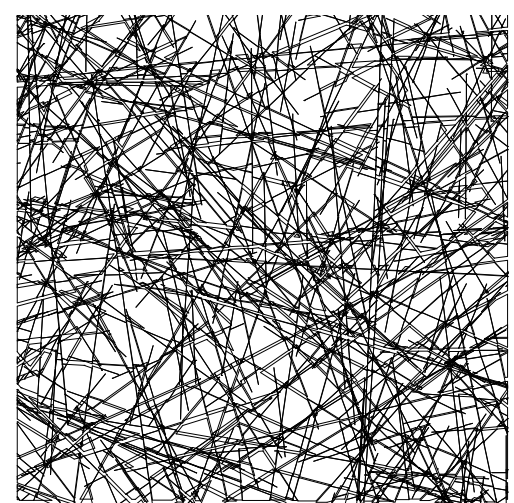

(c) normal distribution, $\theta_{\text {var }}=90^{\circ}$

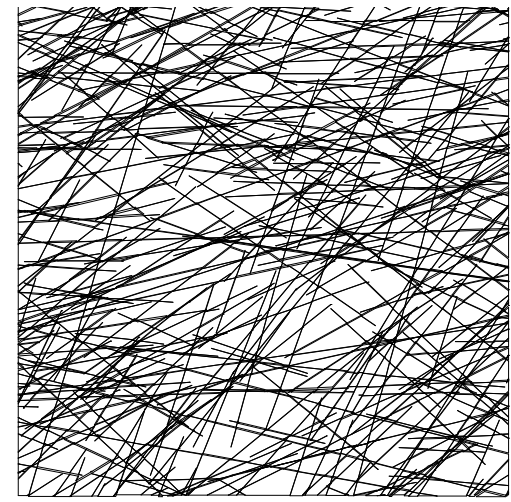

(f) uniform distribution, $\theta_{\text {var }}=90^{\circ}$

Fig. 14 Fracture network models with varying distributions of orientations and corresponding variances. 


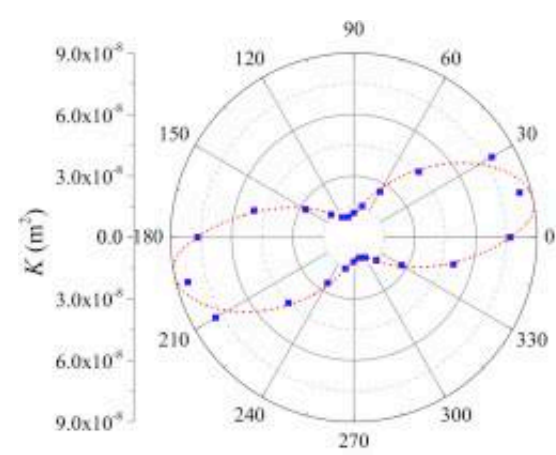

(a) normal distribution, $\theta_{\text {var }}=10^{\circ}$

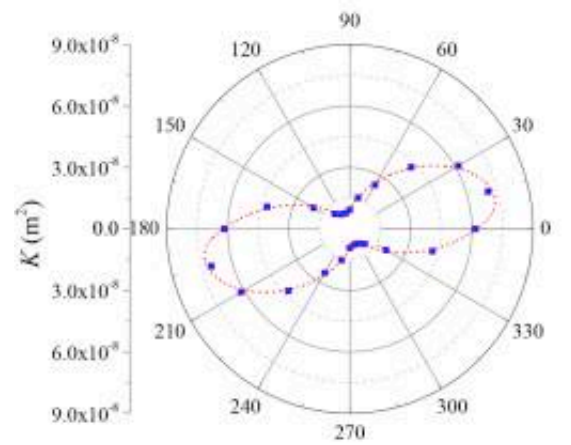

(d) uniform distribution, $\theta_{\text {var }}=10^{\circ}$

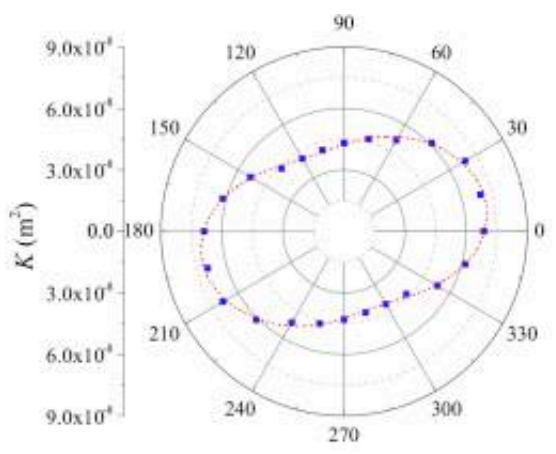

(b) normal distribution, $\theta_{\text {var }}=50^{\circ}$

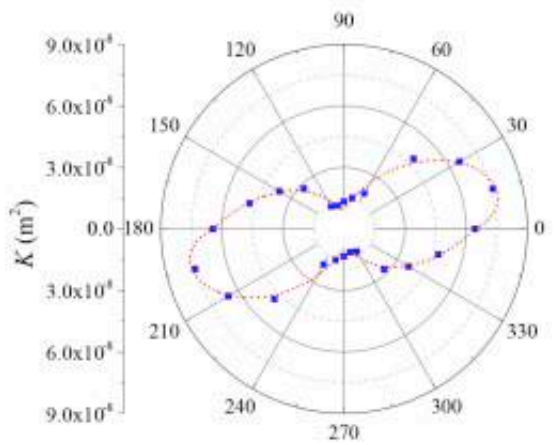

(e) uniform distribution, $\theta_{\text {var }}=50^{\circ}$

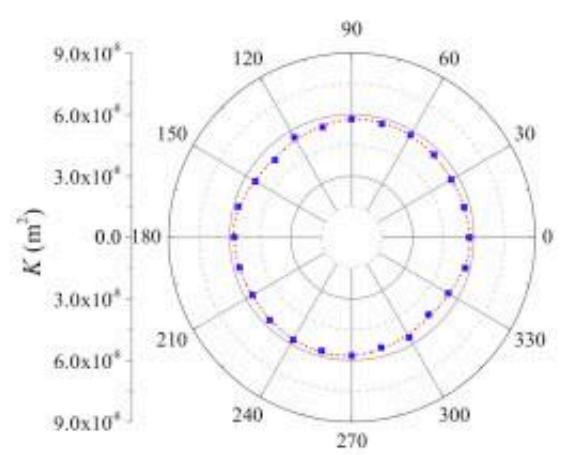

(c) normal distribution, $\theta_{\text {var }}=90^{\circ}$

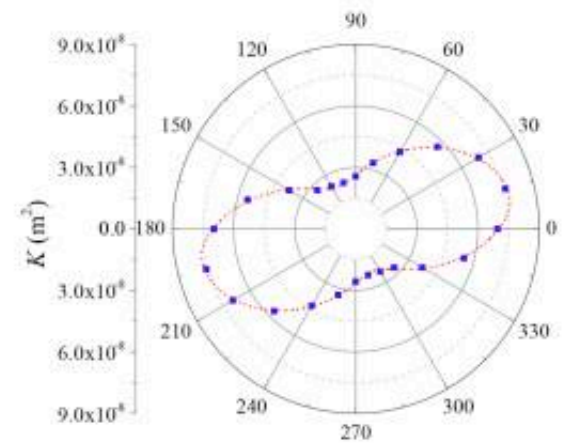

(f) uniform distribution, $\theta_{\text {var }}=90^{\circ}$

Fig. 15 Directional permeability contours with varying distributions of orientations and corresponding variances. 


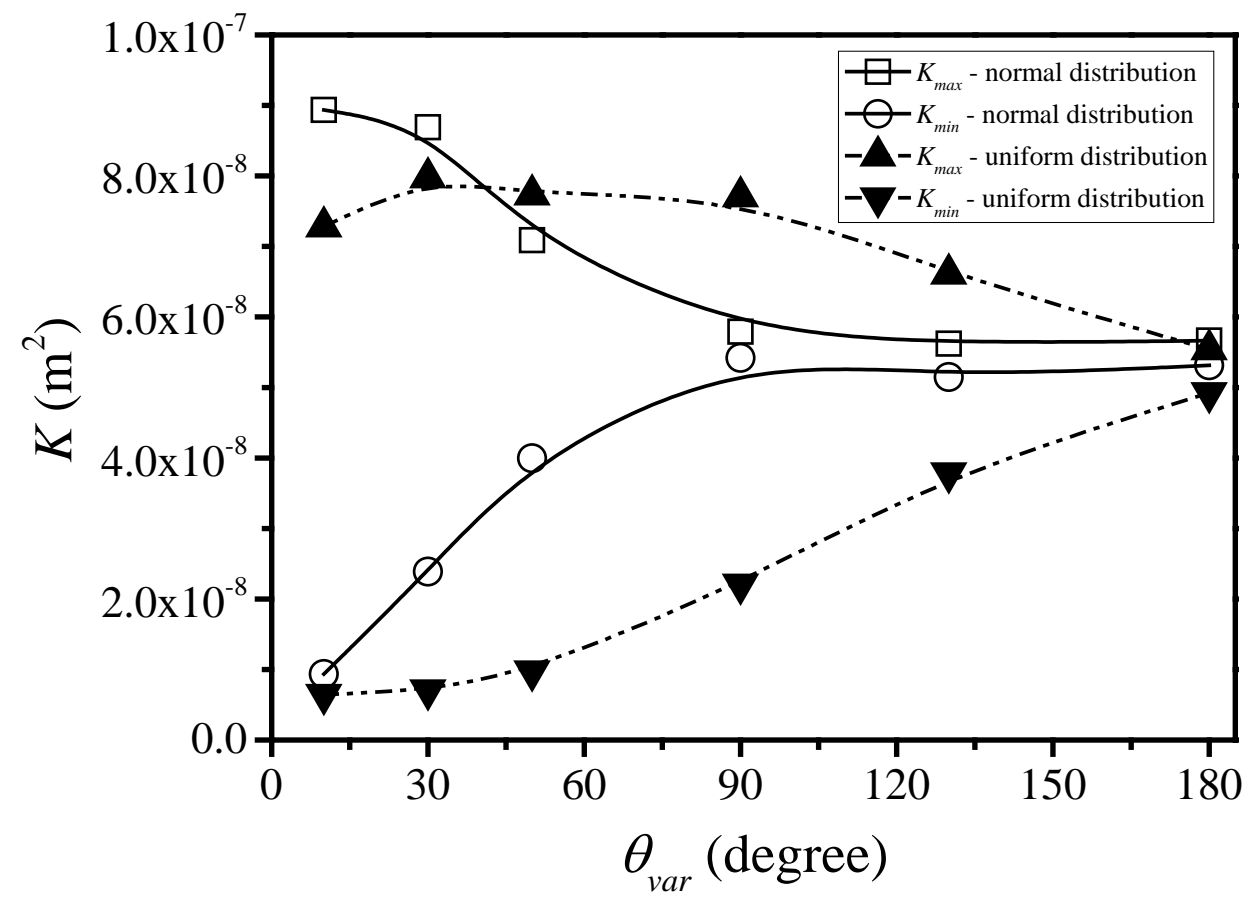

Fig. 16 Variation of permeability with different variances of orientations of the two fracture sets from $10^{\circ}$ to $180^{\circ}$. 


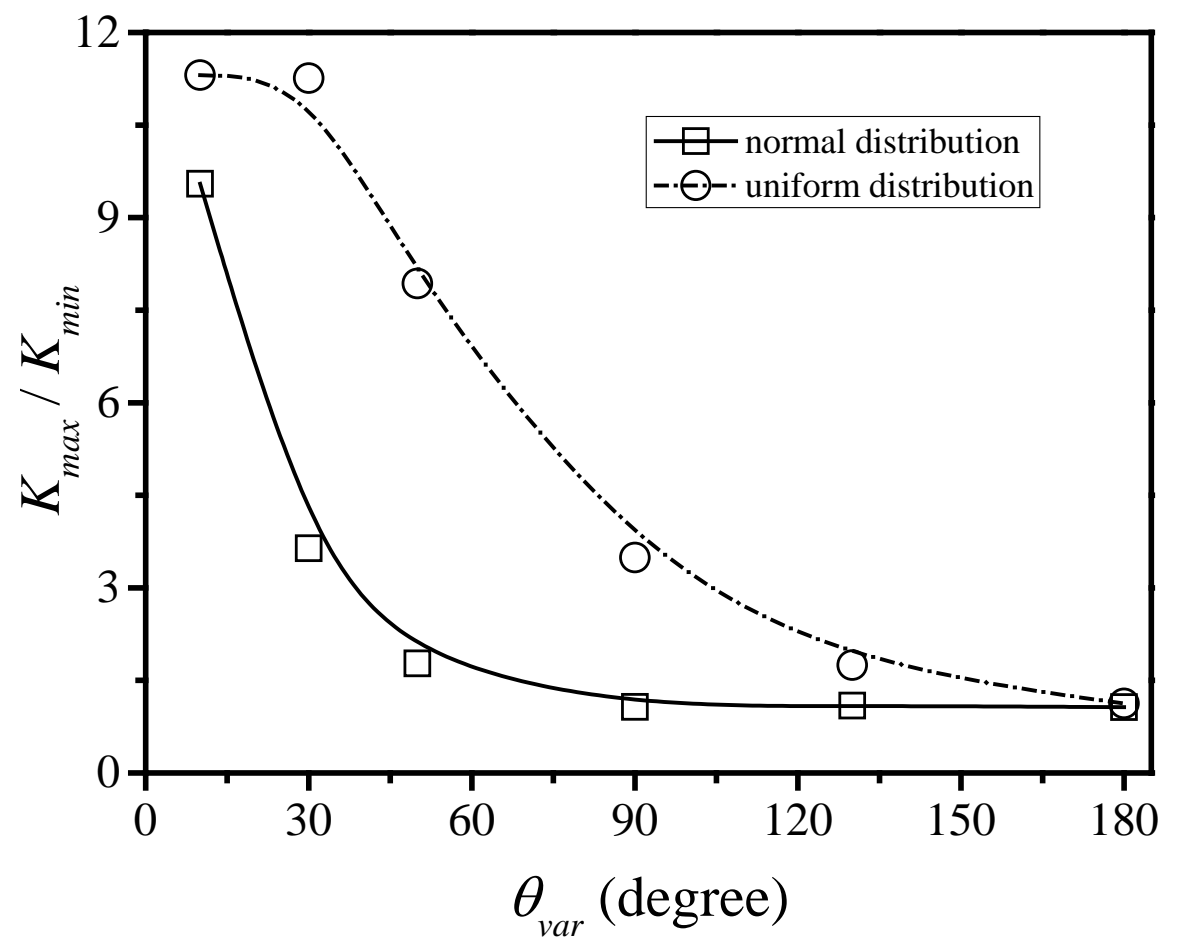

Fig. 17 Relationship between the ratio of the maximum permeability to the minimum permeability and the variance of the orientation of the two fracture sets from $10^{\circ}$ to $180^{\circ}$. 
Table 1 Parameters used for generating fracture networks

\begin{tabular}{|c|c|c|c|c|c|c|c|c|c|c|c|c|c|}
\hline \multirow[b]{2}{*}{$D_{1}$} & \multirow[b]{2}{*}{$D_{2}$} & \multirow[b]{2}{*}{$D_{\mathrm{m}}$} & \multicolumn{2}{|l|}{$e_{1}$} & \multicolumn{2}{|c|}{$e_{2}$} & \multicolumn{2}{|c|}{$\theta_{m}$} & \multicolumn{2}{|c|}{$\theta_{\text {var }}$} & \multirow{2}{*}{$\begin{array}{l}L_{\text {min }} \\
(\mathrm{m})\end{array}$} & \multirow{2}{*}{$\begin{array}{c}L_{\max } \\
(\mathrm{m})\end{array}$} & \multirow{2}{*}{$\begin{array}{c}L_{R E V} \\
(\mathrm{~m})\end{array}$} \\
\hline & & & $\begin{array}{c}\text { Initial value } \\
(\mathrm{mm})\end{array}$ & Range & $\begin{array}{l}\text { Initial value } \\
(\mathrm{mm})\end{array}$ & $\begin{array}{l}\text { Range } \\
(\mathrm{mm})\end{array}$ & Initial value & Range & Initial value & Range & & & \\
\hline 1.3 & 1.3 & 1.3 & 0.065 & - & 0.065 & $0.065 \sim 0.325$ & $30^{\circ}$ & $30 \sim 90^{\circ}$ & $10^{\circ}$ & $10 \sim 180^{\circ}$ & 2.0 & 25.0 & 15 \\
\hline 1.3 & 1.4 & 1.35 & 0.065 & - & 0.065 & $0.065 \sim 0.325$ & $30^{\circ}$ & $30 \sim 90^{\circ}$ & $10^{\circ}$ & $10 \sim 180^{\circ}$ & 1.0 & 13.0 & 9 \\
\hline 1.3 & 1.5 & 1.4 & 0.065 & - & 0.065 & $0.065 \sim 0.325$ & $30^{\circ}$ & $30 \sim 90^{\circ}$ & $10^{\circ}$ & $10 \sim 180^{\circ}$ & 1.0 & 10.0 & 6 \\
\hline 1.4 & 1.4 & 1.4 & 0.065 & - & 0.065 & $0.065 \sim 0.325$ & $30^{\circ}$ & $30 \sim 90^{\circ}$ & $10^{\circ}$ & $10 \sim 180^{\circ}$ & 0.5 & 7.0 & 5 \\
\hline 1.4 & 1.5 & 1.45 & 0.065 & - & 0.065 & $0.065 \sim 0.325$ & $30^{\circ}$ & $30 \sim 90^{\circ}$ & $10^{\circ}$ & $10 \sim 180^{\circ}$ & 0.5 & 5.0 & 4 \\
\hline 1.5 & 1.5 & 1.5 & 0.065 & - & 0.065 & $0.065 \sim 0.325$ & $30^{\circ}$ & $30 \sim 90^{\circ}$ & $10^{\circ}$ & $10 \sim 180^{\circ}$ & 0.25 & 4.0 & 3 \\
\hline
\end{tabular}

